\title{
Relacionamento entre os custos das políticas de manutenção por idade e por bloco
}

\author{
Dulce Ayako Kurauti
}

DISSERTAÇÃO APRESENTADA

$\mathrm{AO}$

INSTITUTO DE MATEMÁTICA E ESTATÍSTICA

DA

UNIVERSIDADE DE SÃO PAULO

PARA OBTENÇÃO DO GRAU DE MESTRE

EM

ESTATÍSTICA

Área de concentração: Probabilidade

Orientador: Prof. Dr. Vanderlei da Costa Bueno

São Paulo, 1.995. 


\section{Agradecimentos}

ao Prof. Dr. Vanderlei da Costa Bueno, pela orientação dedicada e atenciosa,

à Vera, pela cuidadosa revisão desta dissertação,

à minha família e aos meus amigos pela compreensão, paciência e constante incentivo. 


\section{Resumo}

Uma área de grande interesse na Teoria da Confiabilidade é o estudo de políticas de manutenção que objetivam reduzir a ocorrência de falhas em sistemas. Duas políticas de importância particular são a política de manutenção por idade e a política de manutenção por bloco. Nas aplicações, cada modelo probabilístico tem sua própria estrutura de custo e o principal problema é encontrar uma expressão para o custo esperado no decorrer do tempo. Na teoria clássica, os cálculos para as políticas de manutenção por idade e por bloco são feitos separadamente.

Nesta dissertação, consideramos um sistema em manutenção com um mecanismo de custo geral. Estabelecemos um relacionamento entre o custo esperado por unidade de tempo para políticas de manutenção por idade e por bloco e um relacionamento entre o custo total esperado com desconto para estas duas políticas. Usamos também tais relacionamentos para encontrar a política de manutenção ótima, por idade e por bloco, para este modelo geral de manutenção. 


\section{Abstract}

One important area of interest in Reliability Theory is the study of maintenance policies in order to reduce the occurrence of system failure. Two policies of particular importance are the age replacement policy and the block replacement policy. In applications, each probabilistic model has its own cost structure and the main problem is to compute an expression to the expected long-run cost. In the classical theory the calculation for the age replacement policy and for the block replacement policy have always been treated separately.

In this dissertation we consider a very general cost mechanism for a maintained system. We establish a relationship between the long-run cost per unit time for an age and block replacement policies and a relationship between the expected total discounted cost for these two policies. Also we use these relationship to find the optimal age and block replacement policies for this general maintenance model. 


\section{Conteúdo}

Introdução 8

Capítulo 1 - Relação entre os custos esperados por unidade de tempo nas políticas de manutenção por idade e por bloco.

1.1 -O modelo geral...................................................................................... 12

1.2 -O custo operacional esperado por unidade de tempo.........................................13

1.3 -Exemplo................................................................................................ 20

Capítulo 2 - Relação entre os custos esperados com desconto nas políticas de manutenção por idade e por bloco.

2.1 -O custo esperado com desconto para uma política de manutenção por idade.. 30

2.2 -O custo esperado com desconto para uma política de manutenção por bloco. 35

2.3 -Relação entre os custos com desconto .................................................................. 38

2.4 -Outras relą̧ões de custo................................................................................. 41

Capítulo 3- Políticas de manutenção ótimas por idade e por bloco para um modelo geral.

3.1 -A função de custo marginal .............................................................................4

3.2 -Resultados de otimização ...............................................................................53

3.3 -Exemplos ...............................................................................................5

Apêndices

A.1 - Demonstrações referentes ao Capítulo 1 .........................................................66

A.2 - Demonstrações referentes ao Capítulo 2 2.........................................................77

A.3 - Demonstrações referentes ao Capítulo 3 ......................................................... 83

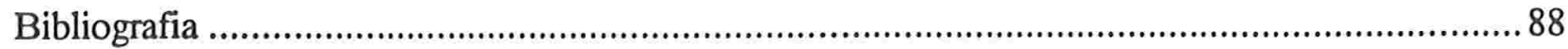


Em muitas situações práticas, a falha de um objeto (de um sistema ou de um componente) em operação pode ser prejudicial. Antes que ocorra tal situação crítica é possível realizar intervenções no intuito de manter o objeto em funcionamento. Uma política de manutenção consiste em reparar e/ou substituir o objeto em determinados instantes, aleatórios ou não, continuamente no tempo. Duas formas padrões de intervenção são as chamadas política de manutenção por idade e política de manutenção por bloco.

Na política de manutenção por idade, uma substituição é realizada sempre que ocorrer uma falha não reparável ou quando o objeto atingir uma idade fixada $T$. Na política de manutenção por bloco, é feita uma substituição sempre que ocorrer uma falha não reparável e a cada unidade T de tempo, isto é, nos tempos $T, 2 T, 3 T, \ldots$, independentemente da idade do objeto. Prova-se que, quando o tempo de vida do objeto tem taxa de falha crescente, intervenções dos tipos acima resultam na diminuição do número de falhas não planejadas.

Fixada a classe de política de manutenção, constrói-se um modelo probabilístico que caracterize uma função de estrutura de custo. Em um intervalo infinito de tempo, uma função de estrutura de custo apropriada é, por exemplo, o custo esperado por unidade de tempo, ou então o custo total esperado com desconto. Uma política de manutenção ótima consiste em encontrar um membro da classe da política da manutenção adotada que minimize a função de estrutura de custo.

Classicamente, as análises para encontrar a política de manutenção ótima dentre as políticas de manutenção por idade e por bloco eram conduzidas separadamente (Barlow, Proschan e Hunter, 1.965). Nesta dissertação, mostraremos que existe um relacionamento adequado entre as funções de estrutura de custo para as duas políticas de manutenção e usaremos esses resultados para achar uma política de manutenção ótima por idade e por bloco em um modelo geral de manutenção. 
No Capítulo 1 do presente trabalho, analisaremos o relacionamento entre a função estrutura de custo para a política de manutenção por idade e por bloco, considerando o custo esperado por unidade de tempo. No Capítulo 2, consideraremos a mesma análise para o custo total esperado com desconto e, no Capítulo 3, procuraremos a política de manutenção ótima por idade e por bloco, usando os resultados obtidos nos capítulos anteriores. 
Capítulo 1

Relação entre os custos esperados por unidade de tempo nas políticas de manutenção por bloco e por idade 


\section{Seção 1.1 - Modelo geral}

Neste capítulo, estudaremos o relacionamento do custo esperado por unidade de tempo entre a política de manutenção por idade e a por bloco, para uma estrutura de custo geral, usando o trabalho de Savits (1.988).

O modelo básico é dado por um processo estocástico $\{R(t), 0 \leq t \leq \zeta\}$, onde $\zeta$ é uma variável aleatória estritamente positiva e com esperança finita. Interpretaremos $\zeta$ como o tempo de vida do objeto caracterizando uma substituição não planejada a um custo $c_{1}$ e $R(t)$ como sendo o custo operacional do objeto no intervalo $[0, t)$.

O mecanismo acima não exclui a possibilidade de falhas no intervalo de tempo $[0, \zeta)$. Em tal caso, assumiremos que a unidade é reparada e que continuará funcionando em algum nível de operação. O custo desses reparos é incorporado no processo $R(t)$. Assim, $\zeta$ é o tempo de uma falha irreparável enquanto que as outras falhas são consideradas reparáveis.

Para reduzir a ocorrência de falhas, adotaremos uma política de manutenção por idade ou uma política de manutenção por bloco. Em qualquer caso, o custo de uma substituição planejada é $c_{2}$.

Em resumo, assumiremos que:

i) $\{R(t), 0 \leq t \leq \zeta\}$ é um processo estocástico não negativo, não decrescente, contínuo à esquerda, com limite à direita e $R(0)=R(0+)=0$.

ii) $R(t+)$ representa o custo operacional da unidade acumulado no intervalo de tempo $[0, t]$, $0 \leq t \leq \zeta$

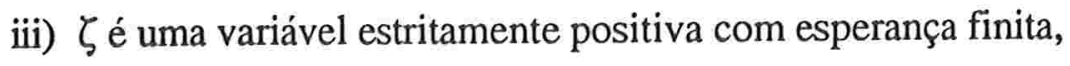

iv) $c_{1}$ é o custo de uma substituição não planejada, $c_{2}$ é o custo de uma substituição planejada e

v) $E[R(t)]<\infty$. 


\section{Seção 1.2 - Custo operacional esperado por unidade de tempo}

Nesta seção, analisaremos o custo operacional total para cada tipo de manutenção e em primeiro lugar, consideraremos a política de manutenção por idade.

Definição 1.2.1: Política de manutenção por idade consiste em substituir um objeto por um novo sempre que ocorrer uma falha não reparável ou quando o objeto atingir uma idade fixada $T$.

Sejam $\left\{R(t), 0 \leq t \leq \zeta_{i}\right\}, i=1,2,3 \ldots$, cópias independentes de $\{R(t), 0 \leq t \leq \zeta\}$.

Introduziremos a seguinte notação:

$\delta_{i}=\min \left(\zeta_{i}, T\right)$

$\xi_{k}= \begin{cases}0, & \text { se } k=0 \mathrm{e} \\ \delta_{1}+\ldots+\delta_{k}, & \text { se } k=1,2, \ldots\end{cases}$

e

$$
R^{*}(t)= \begin{cases}R(t+) & \text { se } 0 \leq t<\delta_{i} \mathrm{e} \\ R\left(\delta_{i}\right)+c_{1} I_{\left\{\zeta_{i}<T\right\}}+c_{2} I_{\left\{\zeta_{i} \geq T\right\}} & \text { se } t \geq \delta_{i}\end{cases}
$$

para $i=1,2, \ldots$

Aqui, $I_{A}$ denota a função indicadora do conjunto $A$.

Se $K_{A}(t ; T)$ denota o custo operacional total em $[0, t]$, é fácil verificar que para $\xi_{k} \leq t \leq \xi_{k+1}, k=0,1, \ldots$

$$
K_{A}(t ; T)=\sum_{i=1}^{k} R^{*}\left(\delta_{i}\right)+R^{*}\left(t-\xi_{k}\right)
$$

Por convenção, uma soma vazia é igual a zero.

O custo esperado total é $E\left[K_{A}(t ; T)\right]$. 
Como os instantes $\delta$ correspondem às renovações do processo concluímos que, assintoticamente, no decorrer do tempo, o custo operacional por unidade de tempo é o custo médio por ciclo dividido pelo comprimento de um ciclo, isto é:

$$
J_{A}(T)=\lim _{t \rightarrow \infty} \frac{E\left[K_{A}(t ; T)\right]}{t}=\frac{E\left[R^{*}(\delta)\right]}{E[\delta]}, \operatorname{com} E\left[\left\|R^{*}(\delta)\right\|<\infty .\right.
$$

O custo médio por ciclo é dado por

$$
A(T)=E\left[R^{*}(\delta)\right]=E\left[R(T)+c_{2} ; T \leq \zeta\right]+E\left[R(\zeta)+c_{1} ; T>\zeta\right]=E\left[K_{A}(\delta ; T)\right]
$$

Consideraremos, no que segue, a política de manutenção por bloco.

Definição 1.2.2: Política de manutenção por bloco é aquela em que ocorre um reparo completo sempre que ocorrer uma falha não reparável e também a cada unidade de tempo $T$, isto é, nos tempos $T, 2 T, 3 T, \ldots$, independentemente da idade do objeto.

Definindo:

$$
\begin{aligned}
& \sigma_{k}= \begin{cases}0, & \text { se } k=0 e \\
\zeta_{1}+\ldots+\zeta_{k}, & \text { se } k=1,2, \ldots\end{cases} \\
& \mathrm{e} \\
& K_{B}(T ; T)= \begin{cases}R(T)+c_{2}, & \text { se } 0<T \leq \sigma_{1} e \\
\sum_{i=1}^{k} R\left(\zeta_{i}\right)+k c_{1}+R\left(T-\sigma_{k}\right)+c_{2}, & \text { se } \sigma_{k}<T \leq \sigma_{k+1}\end{cases}
\end{aligned}
$$

e usando novamente a teoria da renovação, onde as renovações ocorrem nos instantes $T, 2 T$, $3 T, \ldots$, concluímos que, assintoticamente, o custo operacional por unidade de tempo é o custo médio por ciclo dividido pelo tamanho do ciclo, isto é,

$$
J_{B}(T)=\lim _{t \rightarrow \infty} \frac{E\left[K_{B}(t ; T)\right]}{t}=\frac{E\left[K_{B}(T ; T)\right]}{T}, \operatorname{com} E\left[\left|K_{B}(T ; T)\right|\right]<\infty,
$$


onde $K_{B}(t ; T)$ é o custo operacional em $[0, \mathrm{t}]$.

Aqui, o custo médio por ciclo é definido por $B(T)=E\left[K_{B}(T ; T)\right]$.

Nesta seção o nosso principal objetivo é obter uma relaçào matemática entre o custo médio por ciclo de uma política de manutenção por idade e o custo médio por ciclo de uma política de manutenção por bloco, a saber

$$
B(T)=\int_{[0, T)} A(T-x) d U(x)
$$

onde $U(x)=\sum_{k=0}^{\infty} P\left(\sigma_{k} \leq x\right)$ é a função de renovação, gerada pela sequência de variáveis aleatórias independentes e identicamente distribuídas, $\zeta_{1}, \zeta_{2}, \ldots$

Como introdução a este resultado consideremos o seguinte exemplo:

Exemplo 1.2.3:

Consideremos o caso simples em que $R(t) \equiv 0$, onde o único custo provem de trocas planejadas ou não. Se $G$ denota a função de distribuição de $\zeta$ então, para a política de manutenção por idade, o custo médio por ciclo $A(T)$ é dado por

$$
A(T)=c_{1} G(T-)+c_{2} \bar{G}(T-)
$$

onde $\bar{G}(T)=1-G(T)$ denota a probabilidade de sobrevivência e para a política de manutenção por bloco e o custo médio por ciclo $B(T)$ é dado por

$$
B(T)=c_{1} V(T-)+c_{2}
$$

Solução: É fácil notar que na política de manutenção por idade o custo médio por ciclo é

$$
\begin{aligned}
A(T) & =E\left[R^{*}(\delta)\right]=E\left[c_{2} ; T \leq \zeta\right]+E\left[c_{1} ; T>\zeta\right]=c_{2} P(T \leq \zeta)+c_{1} P(T>\zeta)= \\
& =c_{2} P(\zeta \geq T)+c_{1} P(\zeta<T)=c_{2} \bar{G}(T-)+c_{l} G(T-) .
\end{aligned}
$$


No caso da política de manutenção por bloco, além do custo $c_{2}$ correspondente à troca planejada no instante $T$, poderemos ter um número qualquer de trocas não planejadas em $(0, T)$ e portanto o custo médio de um ciclo $B(T)$ é

$$
B(T)=c_{1} \sum_{k=1}^{\infty} P\left(\sigma_{k}<T\right)+c_{2}=c_{1} V(T-)+c 2
$$

onde $V(T-)=\sum_{k=1}^{\infty} P\left(\sigma_{k}<T\right)$ e $\sigma_{k}$ é o instante da k-ésima falha.

Utilizando o Teorema 1.2.4 que define a relação entre os custos médios por ciclo das duas políticas de manutenção, o custo médio por ciclo $B(T)$, para a política de manutenção por bloco, é dado por:

$$
\begin{aligned}
B(T) & =\int_{[O, T)} A(T-x) d U(x)=\int_{[O, T)}\left(c_{l} G((T-x)-)+c_{2} \bar{G}((T-x)-)\right) d U(x)= \\
& =\int_{[O, T)} c_{l}(I-\bar{G}((T-x)-)) d U(x)+\int_{[O, T)} c_{2} \bar{G}((T-x)-) d U(x)= \\
& =c_{l} \int_{[0, T)} d U(x)+\left(c_{2}-c_{l}\right) \int_{[O, T)} \bar{G}((T-x)-) d U(x)= \\
& =c_{l}(U(T-))+\left(c_{2}-c_{l}\right) \int_{[O, T)} \bar{G}((T-x)-) d U(x)= \\
& =c_{l}(U(T-)-l)+c_{2}=c_{l} V(T-)+c_{2} .
\end{aligned}
$$

Na sexta igualdade, usamos o fato de que $\int_{[0 . T]} \bar{G}(T-x) d U(x)=l$ é solução da equação de renovação $I=\bar{G}(T)+\int_{0}^{T} l d G(x)$.

A relação entre $A(T)$ e $B(T)$ é colocada convenientemente no seguinte teorema:

Teorema 1.2.4: No modelo da Seção 1.2, os custos médios por ciclo no caso da política de manutenção por idade $A(T)$ e no caso da política de manutenção por bloco $B(T)$, são relacionados pela seguinte equação:

$$
B(T)=\int_{[O, T)} A(T-x) d U(x) .
$$

onde $U(x)=\sum_{k=0}^{\infty} P\left(\sigma_{k} \leq x\right)$ é a função de renovação, gerada pela sequência de variáveis aleatórias independentes e identicamente distribuídas, $\zeta_{1}, \zeta_{2}, \ldots$ 
Prova:

$$
\begin{aligned}
& B(T)=E\left[K_{B}(T ; T)\right]= \\
= & E\left[K_{B}(T ; T) ; 0<T \leq \sigma_{1}\right]+\sum_{k=1}^{\infty} E\left[K_{B}(T ; T) ; \sigma_{k}<T \leq \sigma_{k+1}\right]= \\
= & E\left[R(T)+c_{2} ; 0<T \leq \sigma_{1}\right]+\sum_{k=1}^{\infty} E\left[\sum_{i=1}^{k} R\left(\zeta_{i}\right)+k c_{1}+R\left(T-\sigma_{k}\right)+c_{2} ; \sigma_{k}<T \leq \sigma_{k+1}\right]= \\
= & \sum_{k=1}^{\infty} \sum_{i=1}^{k} E\left[R\left(\zeta_{i}\right)+c_{1} ; \sigma_{k}<T \leq \sigma_{k+1}\right]+\sum_{k=1}^{\infty} E\left[R\left(T-\sigma_{k}\right)+c_{2} ; \sigma_{k}<T \leq \sigma_{k+1}\right]+ \\
& +E\left[R\left(T-\sigma_{0}\right)+c_{2} ; \sigma_{0}<T \leq \sigma_{1}\right]= \\
= & \sum_{k=1}^{\infty} \sum_{i=1}^{k} E\left[R\left(\zeta_{i}\right)+c_{1} ; \sigma_{k}<T \leq \sigma_{k+1}\right]+\sum_{k=0}^{\infty} E\left[R\left(T-\sigma_{k}\right)+c_{2} ; \sigma_{k}<T \leq \sigma_{k+1}\right]= \\
= & \sum_{i=1}^{\infty} \sum_{k=i}^{\infty} E\left[R\left(\zeta_{i}\right)+c_{1} ; \sigma_{k}<T \leq \sigma_{k+1}\right]+\sum_{k=0}^{\infty} E\left[R\left(T-\sigma_{k}\right)+c_{2} ; \sigma_{k}<T \leq \sigma_{k+1}\right]= \\
= & \sum_{i=1}^{\infty} E\left[R\left(\zeta_{i}\right)+c_{1} ; \sigma_{i}<T\right]+\sum_{k=0}^{\infty} E\left[R\left(T-\sigma_{k}\right)+c_{2} ; \sigma_{k}<T \leq \sigma_{k+1}\right]= \\
= & \sum_{k=0}^{\infty} E\left[R\left(\zeta_{k+1}\right)+c_{1} ; \sigma_{k+1}<T\right]+\sum_{k=0}^{\infty} E\left[R\left(T-\sigma_{k}\right)+c_{2} ; \sigma_{k}<T \leq \sigma_{k+1}\right]
\end{aligned}
$$

A segunda igualdade da expressão acima decorre da definição de $K_{B}(T, T)$ e a quarta pelo Teorema de Fubini.

A primeira parcela da última adição acima pode ser escrita como:

$$
\begin{aligned}
& E\left[R\left(\zeta_{k+1}\right)+c_{1} ; \sigma_{k+1}<T\right]= \\
& =E\left[R\left(\zeta_{k+1}\right)+c_{1} ; \sigma_{k}+\zeta_{k+1}<T\right]= \\
& =E\left[R\left(\zeta_{k+1}\right)+c_{1} ; \sigma_{k}<T, \zeta_{k+1}<T-\sigma_{k}\right]= \\
& =E\left[\left.E\left[R\left(\zeta_{k+1}\right)+c_{1} ; \zeta_{k+1}<T-x\right]\right|_{x=\sigma_{k}} ; \sigma_{k}<T\right]= \\
& =E\left[\left.E\left[R(\zeta)+c_{1} ; \zeta<T-x\right]\right|_{x=\sigma_{k}} ; \sigma_{k}<T\right]
\end{aligned}
$$


A última igualdade se deve ao fato de que $\left\{R(t), 0 \leq t \leq \zeta_{i}\right\}, i=1,2,3 \ldots$, são cópias independentes de $\{R(t), 0 \leq t \leq \zeta\}$.

Como $\sigma_{\kappa+1}=\sigma_{\kappa}+\zeta_{\kappa+1}$, a segunda parcela da última adição acima pode ser escrita como:

$$
\begin{aligned}
& E\left[R\left(T-\sigma_{k}\right)+c_{2} ; \sigma_{k}<T \leq \sigma_{k+1}\right]= \\
& =E\left[R\left(T-\sigma_{k}\right)+c_{2} ; \sigma_{k}<T \leq \sigma_{k}+\zeta_{k+1}\right]= \\
& =E\left[R\left(T-\sigma_{k}\right)+c_{2} ; \sigma_{k}<T, \zeta_{k+1} \geq T-\sigma_{k}\right]= \\
& =E\left[\left.E\left[R(T-x)+c_{2} ; \zeta_{k+1} \geq T-x\right]\right|_{x=\sigma_{k}} ; \sigma_{k}<T\right]= \\
& =E\left[\left.E\left[R(T-x)+c_{2} ; \zeta \geq T-x\right]\right|_{x=\sigma_{k}} ; \sigma_{k}<T\right] .
\end{aligned}
$$

Novamente, a última igualdade se deve ao fato de que $\left\{R(t), 0 \leq t \leq \zeta_{i}\right\}, i=1,2,3 \ldots$, são cópias independentes de $\{R(t), 0 \leq t \leq \zeta\}$.

Então,

$$
\begin{aligned}
& \sum_{k=0}^{\infty} E\left[R\left(\zeta_{k+1}\right)+c_{1} ; \sigma_{k+1}<T\right]= \\
= & \sum_{k=0}^{\infty} \int_{0}^{T} E\left[R(\zeta)+c_{1} ; \zeta<T-x\right] d P\left(\sigma_{k} \leq x\right)= \\
= & \int_{0}^{T} E\left[R(\zeta)+c_{1} ; \zeta<T-x\right] \sum_{k=0}^{\infty} d P\left(\sigma_{k} \leq x\right)= \\
= & \int_{0}^{T} E\left[R(\zeta)+c_{1} ; \zeta<T-x\right] d \sum_{k=0}^{\infty} P\left(\sigma_{k} \leq x\right)= \\
= & \int_{0}^{T} E\left[R(\zeta)+c_{1} ; \zeta<T-x\right] d U(x) . \\
= & \int_{0}^{T} E\left[R(\zeta)+c_{1} ; \zeta<T-x\right] d \sum_{k=0}^{\infty} P\left(\sigma_{k} \leq x\right)= \\
= & \int_{0}^{T} E\left[R(\zeta)+c_{1} ; \zeta<T-x\right] d U(x) \mathrm{e}
\end{aligned}
$$




$$
\begin{aligned}
& \sum_{k=0}^{\infty} E\left[R\left(T-\sigma_{k}\right)+c_{2} ; \sigma_{k}<T \leq \sigma_{k+1}\right]=\sum_{k=0}^{\infty} \int_{0}^{T} E\left[R(T-x)+c_{2} ; \zeta \geq T-x\right] d P\left(\sigma_{k} \leq x\right)= \\
& =\int_{0}^{T} E\left[R(T-x)+c_{2} ; \zeta \geq T-x\right] d \sum_{k=0}^{\infty} P\left(\sigma_{k} \leq x\right)=\int_{0}^{T} E\left[R(T-x)+c_{2} ; \zeta \geq T-x\right] d U(x)
\end{aligned}
$$

onde $U(x)=\sum_{k=0}^{\infty} P\left(\sigma_{k} \leq x\right)$ é a função de renovação, gerada pela sequência de variáveis aleatórias independentes e identicamente distribuídas, $\zeta_{1}, \zeta_{2}, \ldots$

Consequentemente temos que:

$$
\begin{aligned}
B(T) & =\sum_{k=0}^{\infty}\left\{E\left[R\left(\zeta_{k+1}\right)+c_{1} ; \sigma_{k+1}<T\right]+E\left[R\left(T-\sigma_{k}\right)+c_{2} ; \sigma_{k}<T \leq \sigma_{k+1}\right]\right\}= \\
& =\int_{[0, T)}\left\{E\left[R(T-x)+c_{2} ; \zeta \geq T-x\right]+E\left[R(\zeta)+c_{1} ; \zeta<T-x\right]\right\} d U(x)= \\
& =\int_{[0, T)} A(T-x) d U(x)
\end{aligned}
$$

Observamos que algumas vezes, na literatura, a função de renovação $U(x)$ é definida como $U(x)=\sum_{k=1}^{\infty} P\left(\sigma_{k} \leq x\right)$ ao invés de $\sum_{k=0}^{\infty} P\left(\sigma_{k} \leq x\right)$. Para distinguir as duas formas, denotaremos $V(x)=\sum_{k=1}^{\infty} P\left(\sigma_{k} \leq x\right)$. Assim, $V(x)=U(x)-1$ conta o número esperado de renovações em $[0, \mathrm{x}]$ ignorando a renovação inicial em $t=0$. 


\section{Seção 1.3 - Exemplo}

Exemplo 1.3.1: (Reparo mínimo dependente da idade).

Consideremos um sistema de manutenção por idade no qual o objeto em operação é substituído por um novo quando atinge uma idade $T$ a um custo $c_{2}$ (troca planejada). Quando ocorrer uma falha em um instante $y<T$ o objeto será substituído por um novo (troca não planejada) com probabilidade $p(y)$ a um custo $c_{1}$, ou será reparado minimamente com probabilidade $q(y)=1-p(y)$. Um reparo mínimo recupera o objeto nas condições de funcionamento imediatamente anterior a falha. O custo do i-ésimo reparo mínimo na idade $y$ é $c_{o}^{i}(y)$.

Assumiremos que a vida do objeto é descrita por uma função de distribuição $F$ contínua tal que $\bar{F}(t)>0$ para todo $t \geq 0$.

Se somente reparos mínimos são permitidos, o processo de falha é conhecido como um processo pontual $\left\{S_{n}, n \geq 1\right\}$ na reta real $\Re$ cujo processo de contagem correspondente $\{N(t), t \geq 0\}$ é um processo de Poisson não-homogêneo com função de média $\Lambda(t)=E\{N(t)\}=-\ln \bar{F}(t), \mathrm{t} \geq 0$.

Para formalizarmos o nosso exemplo, consideremos uma sequência $\left\{S_{n}, Z_{n} ; n \geq 1\right\}$ de variáveis aleatórias, onde $S_{n} \geq 0$ denota o instante do n-ésimo reparo e $Z_{n}=0$ ou 1 indica um reparo mínimo ou um reparo completo no instante $S_{n-1}$, respectivamente $\left(S_{o} \equiv 0, Z_{1}=0\right)$. Sejam $p: \Re_{+} \rightarrow[0,1]$, uma função mensurável e $q=1-p$ de forma que $P\left\{Z_{n}=1\right\}=p\left(S_{n-1}\right)$ para $n \geq 1$, com $S_{0}=0$.

A distribuição inicial do processo é dada por

$$
P\left\{S_{1}>t, Z_{1}=z\right\}=\bar{F}(t) I_{\{0\}}(z)
$$


e a n-ésima probabilidade de transição é dada por

$$
P\left\{S_{n+1}>t, Z_{n+1}=z / S_{1}, Z_{1}, \ldots, S_{n}, Z_{n}\right\}=p\left(S_{n}\right)^{2} q\left(S_{n}\right)^{1-z} \frac{\bar{F}\left(S_{n} \vee t\right)}{\bar{F}\left(S_{n}\right)}, \text { para } n=1,2, \ldots
$$

Note que, para toda função $f\left(s_{1}, z_{1}, s_{2}, z_{2}, \ldots, s_{n}, z_{n}\right)$ não negativa e mensurável temos

$$
\begin{aligned}
& E\left[f\left(S_{1}, Z_{1}, S_{2}, Z_{2}, \ldots, S_{n}, Z_{n}\right)\right]=\sum_{z_{1}, z_{2}-, z_{n}=0}^{1} \int_{0}^{\infty} \bar{F}^{-1}\left(s_{1}\right) \int_{s_{1}}^{\infty} \bar{F}^{-1}\left(s_{2}\right) \ldots \int_{s_{n-2}}^{\infty} \bar{F}^{-1}\left(s_{n-1}\right) x \\
& \int_{s_{n-1}}^{\infty} \prod_{i=2}^{n} p\left(s_{i-1}\right)^{z_{i}} q\left(s_{i-1}\right)^{z_{i}-1} f\left(s_{1}, 0, s_{2}, z_{2}, \ldots, s_{n}, z_{n}\right) F\left(d s_{n}\right) \ldots F\left(d s_{1}\right) .
\end{aligned}
$$

Enquanto os fracassos são observados, os reparos mínimos são realizados sucessivamente até que ocorra um sucesso ou o objeto atinja a idade $T$, quando um reparo completo é realizado. Assim, o nosso processo geral de falhas se regenera a todo instante em que um reparo completo é realizado. Assumindo que começamos com um objeto novo, os tempos sucessivos de reparos completos formam um processo de renovação.

Como é suficiente modelar o processo de falha até a primeira renovação, consideraremos a análise do processo até o tempo aleatório $S_{v-1}$, onde $v=\inf \left\{n \geq 1: Z_{n}=1\right\}$.

Sejam $N(t)=\sum_{n=1}^{\infty} I_{[0, t]}\left(S_{n}\right)$ o número total de falhas do objeto em $[0, t]$, $L(t)=\sum_{n=1}^{N(t)} Z_{n}$ o número de reparos completos e $M(t)=N(t)-L(t)$ o número de reparos mínimos. 
Para obtermos os resultados deste exemplo, serão necessários os lemas a seguir cujas provas encontram-se no Apêndice A1.

Lema 1.3.1.1: Seja $\left\{\left(S_{n}, Z_{n}\right) ; n \geq 1\right\}$ um processo construído de acordo com a descrição anterior. Se $N(t)=\sum_{n=1}^{\infty} I_{[0, t]}\left(S_{n}\right)$, então $\{N(t), t \geq 0\}$ é um processo de Poisson não homogêneo com função de média $\Lambda(t)=-\ln \bar{F}(t)$.

Prova: Ver demonstração do Lema A.1.1. no Apêndice A1.

Lema 1.3.1.2: Sob as condições do Lema 1.3.1.1., temos que $L(t)=\sum_{n=1}^{N(t)} Z_{n}$, o número de reparos completos e $M(t)=N(t)-L(t)$ o número de reparos mínimos, são processos de Poisson não homogêneos independentes com médias $\int_{0}^{t} p(u) d \Lambda(u) \mathrm{e} \int_{0}^{t} q(u) d \Lambda(u)$ respectivamente, onde $\Lambda(t)=E[N(t)]$

Prova: Ver demonstração do Lema A.1.2. no Apêndice A1.

Corolário 1.3.1.3: $\{M(t), t \geq 0\}$ e $\zeta$ são independentes.

Prova: Como $\zeta=\inf \{t \geq 0, L(t)=1\}$, usando o lema anterior $M(t)$ e $L(t)$ são independentes e o resultado está demonstrado.

Lema 1.3.1.4: $\mathrm{O}$ tempo entre trocas não planejadas $\zeta$ tem função de sobrevivência

$$
\bar{G}(t)=\exp \left\{-\int_{0}^{t} p(y) \bar{F}(y) d F(y)\right\} \equiv \bar{F}_{p}(t)
$$

Prova: Ver demonstração do Lema A.1.3. no Apêndice A1. 
Corolário 1.3.1.5: O custo operacional $R(t), 0 \leq \mathrm{t}<\zeta$, que é a soma dos custos de cada reparo mínimo feito antes de uma renovação, é dado por

$$
R(t+)=\sum_{k=1}^{M(t)} c_{0}^{i}\left(S_{i}\right)
$$

onde $S_{1}, S_{2}, \ldots$ são os tempos de chegada de um processo de Poisson não homogêneo $M(t)$ com função de média $\int_{0}^{t} q(y) \bar{F}^{-1}(y) d F(y)$, onde $\bar{F}^{-1}(y)=\frac{1}{\bar{F}(y)}$.

Prova: O corolário segue do Lema 1.3.1.2.

Neste exemplo a expressão para $A(T)$ é dada por

$$
\begin{aligned}
& A(T)=c_{1} F_{p}(T)+c_{2} \bar{F}_{p}(T)+\int_{0}^{T} q(y) h(y) \bar{F}^{-1}(y) \bar{F}_{p}(y) d F(y), \text { onde } \\
& h(y)=E\left[c_{0}^{M(y)+1}(y)\right] .
\end{aligned}
$$

Para provar este resultado, será necessário o seguinte lema.

Lema 1.3.1.6: Seja $\{M(t), t \geq 0\}$ um Processo de Poisson não-homogêneo com intensidade $\lambda(t)$ e $\Lambda(t)=E[M(t)]=\int_{0}^{t} \lambda(u) d u$. Denotemos por $S_{1}, S_{2}, \ldots$ os tempos de chegadas sucessivos. Assumamos que no instante $S_{i}$, existe um custo $c_{i}\left(S_{i}\right), i=1,2 \ldots$. Se $R(t)$ é o custo total no intervalo $[0, t)$, então

$$
E[R(t)]=\int_{0}^{t} E\left[c_{o}^{M(z)+1}(z)\right] \lambda(z) d z
$$


Prova:

$$
\begin{aligned}
E[R(t)] & =E\left[\sum_{i=1}^{M(t)} c_{0}^{i}\left(S_{i}\right)\right]=\sum_{k=1}^{\infty} E\left[\sum_{i=1}^{k} c_{0}^{i}\left(S_{i}\right) ; S_{k} \leq t<S_{k+1}\right]= \\
& =\sum_{i=1}^{\infty} \sum_{k=i}^{\infty} E\left[c_{0}^{i}\left(S_{i}\right) ; S_{k} \leq t<S_{k+1}\right]=\sum_{i=1}^{\infty} E\left[c_{0}^{i}\left(S_{i}\right) ; S_{i} \leq t\right]= \\
& =\sum_{i=1}^{\infty} E\left[c_{o}^{i}\left(S_{i}\right) ; S_{i} \leq t\right]=\sum_{i=1}^{\infty} \int_{0}^{t} c_{o}^{i}(z) P\left(S_{i} \in d z\right)
\end{aligned}
$$

Como

$$
P\left(S_{i} \leq z\right)=P(M(z) \geq i)=\sum_{k=i}^{\infty} P(M(z)=i)=\sum_{k=i}^{\infty} \exp \{-\Lambda(\mathrm{z})\} \frac{\Lambda(z)^{k}}{k !}
$$

temos

$$
\begin{aligned}
& P\left(S_{i} \in d z\right)=\frac{d\left\{P\left(S_{i} \leq z\right)\right\}}{d z}=\sum_{k=i}^{\infty}\left\{-e^{-\Lambda(z)} \frac{\Lambda^{k}(z)}{k !}+e^{-\Lambda(z)} \frac{k \Lambda^{k-1}(z)}{k(k-1) !}\right\}= \\
& =\sum_{k=i}^{\infty}\left\{-e^{-\Lambda(z)} \frac{\Lambda^{k}(z)}{k !}\right\} d \Lambda(z)+e^{-\Lambda(z)} \frac{\Lambda^{i-1}(z)}{(i-1) !} d \Lambda(z)+\sum_{k=i+1}^{\infty}\left\{e^{-\Lambda(z)} \frac{\Lambda^{k-1}(z)}{(k-1) !}\right\} d \Lambda(z)= \\
& =\sum_{k=i}^{\infty}\left\{-e^{-\Lambda(z)} \frac{\Lambda^{k}(z)}{k !}\right\} d \Lambda(z)+e^{-\Lambda(z)} \frac{\Lambda^{i-1}(z)}{(i-1) !} d \Lambda(z)+\sum_{j=i}^{\infty}\left\{e^{-\Lambda(z)} \frac{\Lambda^{j}(z)}{j !}\right\} d \Lambda(z)= \\
& =e^{-\Lambda(z)} \frac{\Lambda^{i-1}(z)}{(i-1) !} d \Lambda(z)=P\{M(z)=i-1\} d \Lambda(z) .
\end{aligned}
$$

Consequentemente

$$
\begin{aligned}
E[R(t)] & =\sum_{i=1}^{\infty} \int_{0}^{t} c_{0}^{i}(z) P\{M(z)=i-1\} \lambda(z) d z=\sum_{j=0}^{\infty} \int_{0}^{t} c_{0}^{j+1}(z) P\{M(z)=j\} \lambda(z) d z= \\
& =\int_{0}^{t} \sum_{j=0}^{\infty} c_{0}^{j+1}(z) P\{M(z)=j\} \lambda(z) d z=\int_{0}^{t} E\left[c_{0}^{M(z)+1}(z)\right] \lambda(z) d z .
\end{aligned}
$$

Assim, segue o resultado. 
Teorema 1.3.1.7: Sob o modelo acima o custo médio por ciclo em uma política de manutenção por idade é

$$
A(T)=c_{1} F_{p}(T)+c_{2} \bar{F}_{p}(T)+\int_{0}^{T} q(y) h(y) \bar{F}^{-1}(y) \bar{F}_{p}(y) d F(y)
$$

onde $h(y)=E\left[c_{0}^{M(y)+1}\right]$

Prova: Note que $E\left[R^{*}\right]=E\left[R^{*} ; \zeta>T\right]+E\left[R^{*} ; \zeta \leq T\right]$, onde

$$
\begin{aligned}
R^{*} & =I_{(T, \infty)}(\zeta)\left\{R(T)+c_{2}\right\}+I_{[0, T]}(\zeta)\left\{R(\zeta)+c_{1}\right\}= \\
& =I_{(T, \infty)}(\zeta)\left\{\sum_{i=1}^{M(T)} c_{0}^{i}\left(S_{i}\right)+c_{2}\right\}+I_{[0, T]}(\zeta)\left\{\sum_{i=1}^{M(\zeta)} c_{0}^{i}\left(S_{i}\right)+c_{1}\right\}
\end{aligned}
$$

Usando o Lema 1.3.1.6 e a independência entre $\zeta$ e $\{M(t) ; t \geq 0\}$, podemos

escrever

$$
\begin{aligned}
E\left[R^{*} ; \zeta>T\right] & =E\left[I_{(T ; \infty)}(\zeta)\left\{c_{2}+\sum_{i=1}^{M(T)} c_{0}^{i}\left(S_{i}\right)\right\}\right]=P\{\zeta>T\}\left\{c_{2}+E\left[\sum_{i=1}^{M(T)} c_{0}^{i}\left(S_{i}\right)\right]\right\}= \\
& =\bar{F}_{p}(T)\left\{c_{2}+\int_{0}^{T} E\left[c_{0}^{M(z)+1}\right\}(z) r(z) d z\right\}=\bar{F}_{p}(T)\left\{c_{2}+\int_{0}^{T} h(z) q(z) r(z) d z\right\} .
\end{aligned}
$$

Similarmente,

$$
\begin{aligned}
& E\left[R^{*}, \zeta \leq T\right]=E\left[I_{[0, T]}(\zeta)\left\{c_{1}+\sum_{i=1}^{M(\zeta)} c_{0}^{i}\left(S_{i}\right)\right\}=\right. \\
& =P\{\zeta \leq T\} c_{1}+E\left\{\sum_{i=1}^{M(\zeta)} c_{0}^{i}\left(S_{i}\right) \zeta \leq T\right\}= \\
& =P\{\zeta \leq T\} c_{1}+\int_{0}^{T} E\left[\sum_{i=1}^{M(y)} c_{0}^{i}\left(S_{i}\right)\right] P\{\zeta \in d y\}= \\
& =P\{\zeta \leq T\} c_{1}+\int_{0}^{T} \int_{0}^{y} h(z) q(z) r(z) d z P\{\zeta \in d y\}=
\end{aligned}
$$




$$
\begin{aligned}
& =P\{\zeta \leq T\} c_{1}+\int_{0}^{T} \int_{z}^{T} P\{\zeta \varepsilon d y\} h(z) q(z) r(z) d z= \\
& =P\{\zeta \leq T\} c_{1}+\int_{0}^{T}\left(F_{p}(T)-F_{p}(z)\right) h(z) q(z) r(z) d z= \\
& =P\{\zeta \leq T\} c_{1}+\int_{0}^{T}\left(-1+F_{p}(T)+1-F_{p}(z)\right) h(z) q(z) r(z) d z= \\
& =F_{p}(T) c_{1}+\int_{0}^{T}\left(\bar{F}_{p}(z)-\bar{F}_{p}(T)\right) h(z) q(z) r(z) d z
\end{aligned}
$$

Das duas expressões, obtemos

$$
\begin{aligned}
& A(T)=E\left[R^{*}\right]=E\left[R^{*} ; \zeta \leq T\right]+E\left[R^{*} \zeta>T\right]= \\
& =c_{2} \bar{F}_{p}(T)+\bar{F}_{p}(T) \int_{0}^{T} h(z) q(z) r(z) d z+c_{1} F_{p}(T)-\bar{F}_{p}(T) \int_{0}^{T} h(z) q(z) r(z) d z+ \\
& +\int_{0}^{T} \bar{F}_{p}(z) h(z) q(z) r(z) d z=c_{1} F_{p}(T)+c_{2} \bar{F}_{p}(T)+\int_{0}^{T} \bar{F}_{p}(z) h(z) q(z) r(z) d z
\end{aligned}
$$

Teorema 1.3.1.8: Sob o modelo acima o custo médio por ciclo em uma política de manutenção por bloco é

$$
B(T)=\int_{0}^{T} A(T-x) d U(x)=c_{1} V(T)+c_{2}+\int_{0}^{T} V(T-x) q(x) h(x) \bar{F}^{-1}(x) \bar{F}_{p}(x) d F(x)
$$

\section{Prova:}

$$
\begin{aligned}
& B(T)=\int_{[0, T)} A(T-x) d U(x)=\int_{[0, T)}\left\{c_{1} F_{p}(T-x)+c_{2} \bar{F}_{p}(T-x)+\int_{0}^{T-x} q(y) h(y) \bar{F}_{p}(y) \bar{F}^{-1}(y) d F(y)\right\} d U(x)= \\
& =c_{1} \int_{[0, T)}\left(1-\bar{F}_{p}(T-x)\right) d U(x)+c_{2} \int_{[0, T)} \bar{F}_{p}(T-x) d U(x)+\int_{[0, T)}\left\{\int_{0}^{T-x} q(y) h(y) \bar{F}_{p}(y) \bar{F}^{-1}(y) d F(y)\right\} d U(x)= \\
& =c_{1} \int_{[0, T)} d U(x)+\left(c_{2}-c_{1}\right) \int_{[0, T)} \bar{F}_{p}(T-x) d U(x)+\int_{0}^{T}\left[\int_{(0, T-y]} d U(x)\right] q(y) h(y) \bar{F}_{p}(y) \bar{F}^{-1}(y) d F(y)= \\
& =c_{1} U(T-)+\left(c_{2}-c_{1}\right) \int_{[0, T)} \bar{F}_{p}(T-x) d U(x)+\int_{0}^{T}[U(T-y)-U(0)] q(y) h(y) \bar{F}_{p}(y) \bar{F}^{-1}(y) d F(y)= \\
& =c_{1} V(T)+c_{2}+\int_{0}^{T} V(T-y) q(y) h(y) \bar{F}_{p}(y) \bar{F}^{-1}(y) d F(y) .
\end{aligned}
$$


Corolário 1.3.2.9: No caso em que $p(y)=p$ e $c_{0}^{i}(y)=c_{0}$, temos:

$$
\begin{aligned}
& A(T)=\frac{q c_{0}+p c_{1}}{p}\left(1-\bar{F}^{p}(T)\right)+c_{2} \bar{F}^{p}(T) \mathrm{e} \\
& B(T)=\frac{q c_{0}+p c_{1}}{p} V(T)+c_{2} .
\end{aligned}
$$

\section{Prova:}

Se $p(y)=p$, então

$$
\begin{aligned}
& \bar{F}_{p}(t)=\exp \left\{-\int_{0}^{t} p(y) \bar{F}^{-1}(y) d F(y)\right\}=\exp \left\{-p \int_{0}^{t} \bar{F}^{-1}(y) d F(y)\right\}= \\
& =\{\exp \{p \log \bar{F}(t)\}\}=\bar{F}^{p}(t) .
\end{aligned}
$$

Assim,

$$
\begin{aligned}
A(T) & =c_{1}\left(1-\bar{F}_{p}(t)\right)+c_{2} \bar{F}_{p}(t)+\int_{0}^{T} q c_{0} \bar{F}^{-1}(y) \bar{F}_{p}(y) d F(y)= \\
& =c_{1}\left(1-\bar{F}^{p}(t)\right)+c_{2} \bar{F}^{p}(t)+\int_{0}^{T} q c_{0} \bar{F}^{-1}(y) \bar{F}^{p}(y) d F(y)= \\
& =c_{1}+\left(c_{2}-c_{1}\right) \bar{F}^{p}(t)+\frac{q c_{0}}{p} \int_{0}^{T} \bar{F}^{p-1}(y) p d F(y)= \\
& =c_{1}+\left(c_{1}-c_{2}\right) \bar{F}^{p}(t)+\frac{q c_{0}}{p} \int_{0}^{T}(1-F(y))^{p-1} p d F(y)= \\
& =c_{1}+\left(c_{2}-c_{1}\right) \bar{F}_{p}(t)+\frac{q c_{0}}{p} \int_{0}^{T}(1-F(y))^{p-1} p d F(y)= \\
& =c_{1}+\left(c_{2}-c_{1}\right) \bar{F}^{p}(t)+\frac{q c_{0}}{p}\left[(1-F(0))^{p}-(1-F(T))^{p}\right]= \\
& =c_{1}+\left(c_{2}-c_{1}\right) \bar{F}^{p}(t)+\frac{q c_{0}}{p}\left[1-\bar{F}(T)^{p}\right]= \\
& =\frac{c_{0} q+c_{1} p}{p}\left[1-\bar{F}^{p}(T)^{p}\right]+c_{2} \bar{F}^{p}(t) \mathrm{e}
\end{aligned}
$$




$$
\begin{aligned}
B(T) & =c_{1} V(T)+c_{2}+\int_{0}^{T} V(T-x) q c_{0} \bar{F}^{-1}(x) \bar{F}^{p}(x) d F(x)= \\
& =c_{1} V(T)+c_{2}+\int_{0}^{T} \int_{0}^{T-x} d V(y) q c_{0} \bar{F}^{-1}(x) \bar{F}^{p}(x) d F(x)= \\
& =c_{1} V(T)+c_{2}+q c_{0} \int_{0}^{T}\left[\int_{0}^{T-x} d U(y)\right] \bar{F}^{-1}(x) \bar{F}^{p}(x) d F(x)= \\
& =\frac{p c_{1} V(T)}{p}+c_{2}+\frac{q c_{0}}{p} \int_{0}^{T}\left[\int_{0}^{T-y} \bar{F}^{-1}(x) \bar{F}^{p}(x) p d F(x)\right] d U(y)= \\
& =\frac{p c_{1} V(T)}{p}+c_{2}+\frac{q c_{0}}{p} \int_{0}^{T}\left[1-\bar{F}^{p}(T-y)\right] d U(y)= \\
& =\frac{p c_{1} V(T)}{p}+c_{2}+\frac{q c_{0}}{p} \int_{0}^{T} d U(y)-\frac{q c_{0}}{p} \int_{0}^{T} \bar{F}^{p}(T-y) d U(y)= \\
& =\frac{p c_{1} V(T)}{p}+c_{2}+\frac{q c_{0}}{p} U(T)-\frac{q c_{0}}{p}=\frac{p c_{1} V(T)}{p}+c_{2}+\frac{q c_{0}}{p}[U(T)-1]= \\
& =\frac{p c_{1}+q c_{0}}{p} V(T)+c_{2} .
\end{aligned}
$$


Capítulo 2

Relação entre os custos esperados com desconto nas políticas de manutenção por idade e por bloco 
Seção 2.1 - O custo esperado com desconto para uma política de manutenção por idade

Neste capítulo, estabeleceremos uma relação similar à do capítulo anterior entre o custo com desconto para as políticas de manutenção por bloco e por idade, desenvolvido por Chen e Savits (1.988).

Definimos, para $\alpha>0$, o custo com desconto $\alpha$ para uma política de manutenção por idade em $[0, t]$ por

$$
K_{A}^{(\alpha)}(t)=\int_{(0, t]} e^{-\alpha u} d K_{A}(u)
$$

e para uma política de manutenção por bloco como

$$
K_{B}^{(\alpha)}(t)=\int_{(0, t]} e^{-\alpha u} d K_{B}(u)
$$

onde $K_{A}(t)=K_{A}(t, T)$ é o custo operacional total em $[0, t]$ para uma política de manutenção por idade e $K_{B}(t)=K_{B}(t, T)$ é o custo operacional total em $[0, t]$ para uma política de manutenção por bloco. O custo total com desconto $\alpha$ é obtido substituindo-se $(0, t]$ por $(0, \infty)$.

Como no capítulo anterior, o nosso objetivo principal é relacionar o custo médio por ciclo entre uma política de manutenção por idade e uma política de manutenção por bloco.

Teorema 2.1.1: Nas condições do modelo do Capítulo 1, com parâmetros de custo $c_{1}$ e $c_{2}$ não negativos e $R(t)$ um processo não negativo, o custo total esperado com desconto $\alpha$ para a política de manutenção por idade é dado por

$$
\begin{aligned}
& J_{A}^{(\alpha)}(T)=E\left[\int_{(0, \infty)} e^{-\alpha u} d K_{A}(u)\right]=\frac{A^{(\alpha)}(T)}{1-E\left[e^{-\alpha \delta}\right]}, \\
& \text { onde } A^{(\alpha)}(T)=E\left[\int_{(0, \eta]} e^{-\alpha w} d R^{*}(w)\right]
\end{aligned}
$$




\section{Prova:}

$$
\begin{aligned}
& J_{A}^{(\alpha)}(T)=\lim _{t \rightarrow \infty} E\left[K_{A}^{(\alpha)}(t)\right]=\lim _{t \rightarrow \infty} E\left[\int_{(0, t)} e^{-\alpha u} d K_{A}(u)\right]= \\
& =E\left[\lim _{t \rightarrow \infty} \int_{(0, t]} e^{-\alpha u} d K_{A}(u)\right]=E\left\{\lim _{t \rightarrow \infty}\left[\left.e^{-\alpha u} K_{A}(u)\right|_{0} ^{t}+\int_{(0, t]} \alpha e^{-\alpha u} K_{A}(u) d u\right]=\right. \\
& =E\left\{\int_{0}^{\infty} \alpha e^{-\alpha u} K_{A}(u) d u\right\}=\alpha E\left\{\sum_{k=0}^{\infty} \int_{\zeta_{k}}^{\zeta+1} e^{-\alpha u}\left(\sum_{i=1}^{k} R^{*}\left(\delta_{i}\right)+R^{*}\left(u-\zeta_{k}\right)\right) d u\right\} .
\end{aligned}
$$

A terceira igualdade acima segue do Teorema da Convergência Dominada, e quarta igualdade é obtida por integração por partes, fazendo

$$
v=K_{A}(u) \Rightarrow d v=d K_{A}(u) \text { e } s=e^{-\alpha u} \Rightarrow d s=-\alpha e^{-\alpha u} .
$$

Na última igualdade, usamos a definição de $K_{A}(t)$ do Capítulo 1.

Como $\zeta_{k}=\delta_{1}+\delta_{2}+\ldots+\delta_{k} e \zeta_{k+1}=\zeta_{k}+\delta_{k+1}$, fazendo $\omega=u-\zeta_{k}$ temos

$$
\begin{aligned}
& \alpha \sum_{k=0}^{\infty} E\left[\int_{\zeta_{k}}^{\zeta k+1} e^{-\alpha u} R^{*}\left(u-\zeta_{k}\right) d u\right]= \\
& =\alpha \sum_{k=0}^{\infty} E\left[\int_{0}^{\delta_{k+1}} e^{-\alpha\left(\omega+\zeta_{k}\right)} R^{*}(\omega) d \omega\right]= \\
& =\alpha \sum_{k=0}^{\infty} E\left[e^{-\alpha \zeta_{k}} \int_{0}^{\delta_{k+1}} e^{-\alpha \omega} R^{*}(\omega) d \omega\right]= \\
& =\alpha \sum_{k=0}^{\infty} E\left[e^{-\alpha \zeta_{k}}\right] E\left[\int_{0}^{\delta_{k+1}} e^{-\alpha \omega} R^{*}(\omega) d \omega\right]= \\
& =\alpha \sum_{k=0}^{\infty} E\left[e^{-\alpha\left(\delta_{1}+\ldots+\delta_{k}\right)}\right] E\left[\int_{0}^{\delta_{k+1}} e^{-\alpha \omega} R^{*}(\omega) d \omega\right]= \\
& =\alpha \sum_{k=0}^{\infty} E\left[e^{-\alpha \delta_{1}} e^{-\alpha \delta_{2}} \ldots e^{-\alpha \delta_{k}}\right] E\left[\int_{0}^{\delta_{k+1}} e^{-\alpha \omega} R^{*}(\omega) d \omega\right]=
\end{aligned}
$$




$$
\begin{aligned}
& =\alpha \sum_{k=0}^{\infty} \prod_{i=1}^{k} E\left[e^{-\alpha \delta_{i}}\right] E\left[\int_{0}^{\delta_{k+1}} e^{-\alpha \omega} R^{*}(\omega) d \omega\right]= \\
& =\alpha \sum_{k=0}^{\infty} \prod_{i=1}^{k} E\left[e^{-\alpha \delta}\right] E\left[\int_{0}^{\eta} e^{-\alpha \omega} R^{*}(\omega) d \omega\right]= \\
& =\alpha E\left[\int_{0}^{\delta} e^{-\alpha \omega} R^{*}(\omega) d \omega\right] \sum_{k=0}^{\infty}\left(E\left[e^{-\alpha \delta}\right]\right)^{k}= \\
& =\frac{\alpha}{1-E\left[e^{-\alpha \delta}\right]} E\left[\int_{0}^{\delta} e^{-\alpha \omega} R^{*}(\omega) d \omega\right]
\end{aligned}
$$

A sétima igualdade da última expressão decorre da independência dos $\delta_{i}$ 's e a oitava segue do fato que os $\delta_{i}$ 's são identicamente distribuídos.

Temos também:

$$
\begin{aligned}
\alpha & \sum_{k=0}^{\infty} E\left[\int_{\zeta_{k}}^{\zeta_{k+1}} e^{-\alpha u}\left[\sum_{i=1}^{k} R^{*}\left(\delta_{i}\right)\right) d u\right]=\alpha \sum_{k=0}^{\infty} \sum_{i=1}^{k} E\left[\int_{\zeta_{k}}^{\zeta_{k+1}} e^{-\alpha u} R^{*}\left(\delta_{i}\right) d u\right]= \\
= & \alpha \sum_{i=1}^{\infty} \sum_{k=i}^{\infty} E\left[\int_{\zeta_{k}}^{\zeta_{k+1}} e^{-\alpha u} R^{*}\left(\delta_{i}\right) d u\right]=\alpha \sum_{i=1}^{\infty} E\left[R^{*}\left(\delta_{i}\right) \sum_{k=i}^{\infty} \int_{\zeta_{k}}^{\zeta_{k+1}} e^{-\alpha u} d u\right]= \\
= & \sum_{i=1}^{\infty} E\left[R^{*}\left(\delta_{i}\right) \int_{\zeta i}^{\infty} \alpha e^{-\alpha u} d u\right]=\sum_{i=1}^{\infty} E\left[R^{*}\left(\delta_{i}\right) e^{-\alpha \zeta i}\right]= \\
= & \sum_{i=1}^{\infty} E\left[R^{*}\left(\delta_{i}\right) e^{-\alpha\left(\delta_{1}+\ldots+\delta_{i}\right)}\right]=\sum_{i=1}^{\infty} E\left[R^{*}\left(\delta_{i}\right) e^{-\alpha \delta_{i}} e^{-\alpha\left(\delta_{1}+\ldots+\delta_{i-1}\right)}\right]= \\
= & \sum_{i=1}^{\infty} E\left[R^{*}\left(\delta_{i}\right) e^{-\alpha \delta_{i}}\right] E\left[e^{-\alpha\left(\delta_{1}+\ldots+\delta_{i-1}\right)}\right]=\sum_{i=1}^{\infty} E\left[R^{*}(\delta) e^{-\alpha \eta}\right] E\left[e^{-\alpha\left(\delta_{1}+\ldots+\delta_{i-1}\right)}\right]= \\
= & E\left[R^{*}(\delta) e^{-\alpha \delta}\right] \sum_{j=0}^{\infty} E\left[e^{-\alpha\left(\delta_{1}+\ldots+\delta_{j}\right)}\right]=E\left[R^{*}(\delta) e^{-\alpha \delta}\right] \sum_{j=0}^{\infty}\left(E\left[e^{-\alpha \delta}\right]\right)^{j}= \\
= & \frac{E\left[R^{*}(\delta) e^{-\alpha \delta}\right]}{1-E\left[e^{-\alpha \delta}\right]} .
\end{aligned}
$$


Consequentemente,

$$
\begin{aligned}
& J_{A}^{(\alpha)}(T)=\left(1-E\left[e^{-\alpha \delta}\right]\right)^{-1}\left\{E\left[\left[e^{-\alpha \delta} R^{*}(\delta)\right]+\alpha \int_{0}^{\delta} e^{-\alpha \omega} R^{*}(\omega) d \omega\right]\right\}= \\
& =\left(1-E\left[e^{-\alpha \delta}\right]\right)^{-1} E\left[\int_{0}^{\delta} e^{-\alpha \omega} d R^{*}(\omega)\right]= \\
& =\frac{E\left[\int_{0}^{\delta} e^{-\alpha \omega} d R^{*}(\omega)\right]}{\left(1-E\left[e^{-\alpha \delta}\right]\right)} .
\end{aligned}
$$

Desta maneira demonstramos o teorema.

É fácil notar que os instantes $\eta$ correspondem às renovações de um Processo de Recompensa e que assintoticamente, no decorrer do tempo, o custo médio por unidade de tempo é o custo esperado por ciclo sobre o tamanho esperado de cada ciclo, isto é, $\frac{E\left[R^{*}(\delta)\right]}{E[\delta]}=J_{A}(T)$.

Podemos relacionar esta quantidade com $J_{A}{ }^{(\alpha)}(T)$ obtida no Teorema 2.1.1 através do Lema 2.1.2 e Corolário 2.1.3 que seguem.

Lema 2.1.2: Sob as condições do Teorema 2.1.1, temos que $1-E\left[e^{\alpha \delta}\right]=\alpha \int_{0}^{T} \bar{G}(u) e^{-\alpha u} d u$ onde $\bar{G}(x)=P\{\zeta>x\}$ é a função de sobrevivência de $\zeta$.

\section{Prova:}

Note que

$$
\begin{aligned}
& F_{8}(x)=P(\delta \leq x)=P(\min (\zeta, T) \leq x)=1-P(\min (\zeta, T)>x), \text { isto é, } \\
& \bar{F}_{8}(x)=\left\{\begin{array}{l}
0 \text { se } x \geq T \\
\bar{G}(x) \text { se } x<T .
\end{array}\right.
\end{aligned}
$$

Assim, usando o Teorema de Fubini, 


$$
\begin{aligned}
1-E\left(e^{-\alpha \delta}\right) & =1-\int_{0}^{T} \int_{x}^{\infty}\left(\alpha e^{-\alpha t} d t\right) d F_{\delta}(x)=1-\left[\int_{0}^{T} F_{\delta}(t) \alpha e^{-\alpha t} d t+\int_{T}^{\infty} F_{\delta}(T) \alpha e^{-\alpha t} d t\right]= \\
& =\int_{0}^{\infty} \alpha e^{-\alpha t} d t-\int_{0}^{T} F_{\delta}(t) \alpha e^{-\alpha t} d t-\int_{T}^{\infty} F_{\delta}(T) \alpha e^{-\alpha t} d t= \\
& =\int_{0}^{T} \alpha e^{-\alpha t}\left(1-F_{\delta}(t)\right) d t+\int_{T}^{\infty} \alpha e^{-\alpha t}\left(1-F_{\delta}(T)\right) d t= \\
& =\int_{0}^{T} \alpha e^{-\alpha t} \bar{F}_{\delta}(t) d t=\int_{0}^{T} \alpha e^{-\alpha t} \bar{G}(t) d t .
\end{aligned}
$$

Corolário 2.1.3: Nas condições do Teorema 2.1.1, temos :

$$
J_{A}(T)=\lim _{\alpha \downarrow 0} \alpha J^{\alpha}(T)
$$

Prova: Observe que

$$
\begin{aligned}
& \lim _{\alpha \downarrow 0} \alpha J^{\alpha}(T)=\lim _{\alpha \downarrow 0} \frac{\alpha A^{\alpha}(T)}{\alpha \int_{0}^{T} \bar{G}(u) e^{-\alpha u} d u}= \\
& \lim _{\alpha \downarrow 0} \frac{A^{\alpha}(T)}{\int_{0}^{T} \bar{G}(u) e^{-\alpha u} d u}=\lim _{\alpha \downarrow 0} \frac{E\left[\int_{(0, \delta]} e^{-\alpha \omega} d R^{*}(\omega)\right]}{T} \overline{\int_{0}^{T} \bar{G}(u) e^{-\alpha u} d u}= \\
& =\frac{E\left[\int_{(0, \delta]} d R^{*}(\omega)\right]}{\int_{0}^{T} \bar{G}(u) d u}=\frac{E\left[R^{*}(\delta)\right]}{E[\delta]} .
\end{aligned}
$$

Assim, $J_{A}(T)=\frac{E\left[R^{*}(\delta)\right]}{E[\delta]}=\lim _{\alpha \downarrow 0} \alpha J^{\alpha}(T)$. 
Seção 2.2 - O custo esperado com desconto para uma política de manutenção por

\section{bloco}

Agora consideraremos a política de manutenção por bloco. O custo esperado com desconto $\alpha$ para essa política é

$$
J_{B}^{(\alpha)}(T)=\lim _{t \rightarrow \infty} E\left[K_{B}^{(\alpha)}(t)\right]=\lim _{t \rightarrow \infty} E\left[\int_{(0, t]} e^{-\alpha u} d K_{B}(u)\right]
$$

Como no capítulo anterior,

$$
\sigma_{k}=\left\{\begin{array}{l}
0 \mathrm{se} k=0 \mathrm{e} \\
\zeta_{1}+\zeta_{2}+\ldots+\zeta_{k} \text { se } k=1,2, \ldots
\end{array}\right.
$$

Definimos

$$
Q(t)=\left\{\begin{array}{l}
R_{i}(t) \text { se } 0 \leq t \leq \sigma_{1}, \\
\sum_{i=1}^{k} R\left(\zeta_{i}\right)+k c_{1}+R\left(t-\sigma_{k}\right) \text { se } \sigma_{k} \leq t \leq \sigma_{k+1}
\end{array}\right.
$$

e consideramos $\left\{Q_{i}(t), t \geq 0\right\}, i=1,2, \ldots$, cópias independentes de $\{Q(t), \mathrm{t} \geq 0\}$.

Definindo

$$
Q_{i}^{*}(t)=\left\{\begin{array}{l}
Q_{i}(t+) \text { se } 0 \leq t<T \text { e } \\
Q_{i}(T)+c_{2} \text { se } t \geq T
\end{array}\right.
$$

podemos escrever

$$
K_{B}(t)=\sum_{i=1}^{k} Q_{i}^{*}(T)+Q_{k+1}^{*}(t-k T) \text { se } k T \leq t<(k+1) T, k=0,1,2, \ldots
$$

Uma expressão para o custo esperado com desconto $\alpha$ para a política de manutenção por bloco é obtida pelo seguinte teorema: 
Teorema 2.2.1: Nas condições do modelo do Capítulo 1, com parâmetros de custo $c_{1}$ e $c_{2}$ não negativos e $R(t)$ um processo não negativo, o custo total esperado com desconto $\alpha$ para a política de manutenção por bloco é dado por

$$
\begin{aligned}
& J_{B}^{(\alpha)}(T)=\frac{B^{(\alpha)}(T)}{1-e^{-\alpha T}} \\
& \text { onde } B^{(\alpha)}(T)=E\left[\int_{(0, T]}^{-\alpha \omega} d Q^{*}(\omega)\right] .
\end{aligned}
$$

Prova: Observe que

$$
\begin{aligned}
J_{B}^{(\alpha)}(T) & =\lim _{t \rightarrow \infty} E\left[K_{B}^{(\alpha)}(t)\right]=\lim _{t \rightarrow \infty} E\left[\int_{(0, t]} e^{-\alpha u} d K_{B}(u)\right]=E\left[\int_{0}^{\infty} e^{-\alpha u} d K_{B}(u)\right]= \\
& =E\left[-\left.e^{-\alpha u} K_{B}(u)\right|_{0} ^{\infty}+\alpha \int_{0}^{\infty} e^{-\alpha u} K_{B}(u) d u\right]=E\left[\alpha \int_{0}^{\infty} e^{-\alpha u} K_{B}(u) d u\right]= \\
& =\alpha E\left[\sum_{k=1}^{\infty} \int_{k T}^{(k+1) T} e^{-\alpha u}\left(\sum_{i=1}^{k} Q_{i}^{*}(T)+Q_{k+1}^{*}(u-k T)\right) d u+\int_{0}^{T} e^{-\alpha u} Q_{1}^{*}(u) d u\right]= \\
& =\alpha E\left[\sum_{k=1}^{\infty} \int_{0}^{T} e^{-\alpha(k T+\mathrm{v})}\left(\sum_{i=1}^{k} Q_{i}^{*}(T)+Q_{k+1}^{*}(v)\right) d v+\int_{0}^{T} e^{-\alpha v} Q_{1}^{*}(v) d v\right]= \\
& =\alpha E\left[\left[\sum_{k=1}^{\infty} \int_{0}^{T} e^{-\alpha(k T+v)}\left(\sum_{i=1}^{k} Q_{i}^{*}(T)\right) d v+\sum_{k=0}^{\infty} \int_{0}^{T} e^{-\alpha(k T+v)} Q_{k+1}^{*}(v) d v\right]\right]
\end{aligned}
$$

A terceira igualdade acima segue do Teorema da Covergência Dominada e a quarta por integração por partes fazendo $u-k T=v \Rightarrow u=k T+v$.

Considerando cada termo separadamente, temos que a primeira parcela da última adição da expressão acima pode ser escrita como

$$
\begin{aligned}
& E\left[\sum_{k=1}^{\infty} e^{-k T \alpha} \sum_{i=1}^{k} Q_{i}^{*}(T) \int_{0}^{T} \alpha e^{-\alpha v} d v\right]=E\left[\sum_{k=1}^{\infty} e^{-\alpha k T} \sum_{i=1}^{k} Q_{i}^{*}(T)\left(1-e^{-\alpha T}\right)\right]= \\
& =\left(1-e^{-\alpha T}\right) \sum_{k=1}^{\infty} e^{-\alpha k T} \sum_{i=1}^{k} E\left[Q_{i}^{*}(T)\right]=\left(1-e^{-\alpha T}\right) \sum_{k=1}^{\infty} e^{-\alpha k T} \sum_{i=1}^{k} E\left[Q^{*}(T)\right]= \\
& =\left(1-e^{-\alpha T}\right) \sum_{k=1}^{\infty} e^{-\alpha k T} k E\left[Q^{*}(T)\right]=\frac{\left(1-e^{-\alpha T}\right) E\left[Q^{*}(T)\right] e^{-\alpha T}}{\left(1-e^{-\alpha T}\right)^{2}}=\frac{E\left[Q^{*}(T)\right] e^{-\alpha T}}{\left(1-e^{-\alpha T}\right)} .
\end{aligned}
$$


E a segunda parcela pode ser escrita como

$$
\begin{aligned}
& \alpha E\left[\sum_{k=0}^{\infty} \int_{0}^{T} e^{-\alpha(k T+v)} Q_{k+1}^{*}(v) d v\right]=\sum_{k=0}^{\infty} e^{-\alpha k T} E\left[\int_{0}^{T} \alpha e^{-\alpha v} Q_{k+1}^{*}(v) d v\right]= \\
& =\sum_{k=0}^{\infty} e^{-\alpha k T} E\left[-\left.e^{-\alpha v} Q_{k+1}^{*}(v)\right|_{0} ^{T}+\int_{0}^{T} e^{-\alpha v} d Q_{k+1}^{*}(v)\right]= \\
& =\sum_{k=0}^{\infty} e^{-\alpha k T} E\left[-e^{-\alpha T} Q_{k+1}^{*}(T)+\int_{0}^{T} e^{-\alpha v} d Q_{k+1}^{*}(v)\right]= \\
& =-e^{-\alpha T} \sum_{k=0}^{\infty} e^{-\alpha k T} E\left[Q^{*}(T)\right]+\sum_{k=0}^{\infty} e^{-\alpha k T} E\left[\int_{0}^{T} e^{-\alpha v} d Q^{*}(v)\right]= \\
& =\frac{-e^{-\alpha T} E\left[Q^{*}(T)\right]+E\left[\int_{0}^{T} e^{-\alpha v} d Q^{*}(v)\right]}{1-e^{-\alpha T}}
\end{aligned}
$$

Facilmente obtemos o resultado desejado.

Como anteriormente, temos um Processo de Recompensa onde as renovações ocorrem no instante $T$ e assintoticamente no decorrer do tempo, o custo médio por unidade de tempo é dado por $\frac{E\left[Q^{*}(T)\right]}{T}=J_{B}(T)$, isto é, a razão do custo médio por ciclo e o tamanho médio de um ciclo.

Relacionamos esta quantidade com $J_{B}^{(\alpha)}(T)$, obtida pelo Teorema 2.2.1, através da seguinte igualdade:

$$
J_{B}(T)=\frac{E\left[Q^{*}(T)\right]}{T}=\lim _{\alpha \downarrow 0} \alpha J_{B}^{(\alpha)}(T)
$$


Corolário 2.2.2 : Nas condições do Teorema 2.2.1, temos

$$
J_{B}(T)=\lim _{\alpha\rfloor 0} \alpha J_{B}^{(\alpha)}(T)
$$

Prova: Note que

$$
\begin{aligned}
\lim _{\alpha \rightarrow 0} \alpha J_{B}^{(\alpha)} & =\lim _{\alpha \rightarrow 0} \frac{\left(\alpha E\left[\int_{0}^{T} e^{-\alpha \omega} d Q^{*}(\omega)\right]\right)^{\prime}}{\left(1-e^{-\alpha T}\right)^{\prime}}= \\
& =\lim _{\alpha \rightarrow 0} \frac{\left(E\left[\int_{0}^{T} e^{-\alpha \omega} d Q^{*}(\omega)\right]-\alpha E\left[\int_{0}^{T} \omega e^{-\alpha \omega} d Q^{*}(\omega)\right]\right)}{T e^{-\alpha T}}= \\
& =\frac{E\left[\int_{0}^{T} d Q^{*}(\omega)\right]}{T}=\frac{E\left[Q^{*}(T)\right]}{T} .
\end{aligned}
$$

\section{Seção 2.3 - Relação entre os custos com desconto}

Para estabelecer a relação fundamental entre $A^{(\alpha)}(T)$ e $B^{(\alpha)}(T)$, os custos totais esperados referentes à política de manutenção por idade e à política de manutenção por bloco, respectivamente, procederemos como no Capítulo 1. Para tanto, utilizaremos os dois lemas seguintes cujas provas estão no Apêndice.

Lema 2.3.1 : Nas condições do Teorema 2.2.1., temos

$$
\begin{aligned}
\int_{(o, T]}^{e^{-\alpha \omega} d Q^{*}(\omega)} & =\sum_{j=1}^{k}\left[e^{-\alpha \sigma_{j}-1} R^{(\alpha)}\left(\zeta_{j}\right)+e^{-\alpha \sigma_{j}} c_{1}\right]+e^{-\alpha \sigma_{k}} R^{(\alpha)}\left(T-\sigma_{k}\right)+e^{-\alpha T} c_{2} \quad \text { onde } \\
R^{(\alpha)}(t) & =\alpha \int_{0}^{t} e^{-\alpha v} R(v+) d v+e^{-\alpha t} R(t) \text { para } \sigma_{k}<t \leq \sigma_{k+1} .
\end{aligned}
$$

Podemos pensar em $R^{(\alpha)}(t)$ como o custo operacional com desconto $\alpha$ da i-ésima unidade em funcionamento no intervalo de tempo $[0, t)$.

Prova: Ver demonstração do Lema A.2.1 do Apêndice A2. 
Lema 2.3.2: Nas condições do Teorema 2.1.1.,

$$
A^{(\alpha)}(T)=E\left[\left(e^{-\alpha \zeta} c_{1}+R^{(\alpha)}(\zeta)\right) ; \zeta<T\right]+E\left[\left(e^{-\alpha T} c_{2}+R^{(\alpha)}(T)\right) ; \zeta \geq T\right]
$$

Prova: Ver demonstração do Lema A.2.2 do Apêndice A2.

Teorema 2.3.3:O custo esperado com desconto $\alpha$ da política de manutenção por bloco, $B^{(\alpha)}(T)$, pode ser escrito em função de $A^{(\alpha)}(T)$

$$
B^{(\alpha)}(T)=\int_{[0, T)} e^{-\alpha x} A^{(\alpha)}(T-x) d U(x)
$$

Prova: Utilizando o Lema 2.3.1, temos

$$
\begin{aligned}
& B^{(\alpha)}(T)=E\left[\int_{(0, T]} e^{\alpha \omega} d Q^{*}(\omega)\right]=\sum_{k=0}^{\infty} E\left[\int_{(0, T]} e^{\alpha \omega} d Q^{*}(\omega) ; \sigma_{k}<T \leq \sigma_{k+1}\right]= \\
& =E\left[e^{-\alpha \sigma_{0}} R\left(T-\sigma_{0}\right)+e^{-\alpha T} c_{2} ; \sigma_{0}<T \leq \sigma_{1}\right]+ \\
& +\sum_{k=1}^{\infty} E\left\{\sum_{j=1}^{k}\left[e^{-\alpha \sigma_{j-1}} R^{(\alpha)}\left(\zeta_{j}\right)+e^{-\alpha \sigma_{j}} c_{l}\right]+e^{-\alpha \sigma_{k}} R^{(\alpha)}\left(T-\sigma_{k}\right)+e^{-\alpha T} c_{2} ; \sigma_{k}<T \leq \sigma_{k+1}\right\}= \\
& =E\left[R(T)+e^{-\alpha T} c_{2} ; \sigma_{0}<T \leq \sigma_{1}\right]+ \\
& +\sum_{j=1}^{\infty} E\left\{\sum_{k=j}^{\infty}\left[e^{-\alpha \sigma_{j-1}} R^{(\alpha)}\left(\zeta_{j}\right)+e^{-\alpha \sigma_{j}} c_{1}\right] ; \sigma_{k}<T \leq \sigma_{k+1}\right\}+ \\
& +\sum_{k=1}^{\infty} E\left\{e^{-\alpha \sigma_{k}} R^{(\alpha)}\left(T-\sigma_{k}\right)+e^{-\alpha T} c_{2} ; \sigma_{k}<T \leq \sigma_{k+1}\right\}= \\
& =\sum_{j=1}^{\infty} E\left\{e^{-\alpha \sigma_{j-1}} R^{(\alpha)}\left(\zeta_{j}\right)+e^{-\alpha \sigma_{j}} c_{l} ; \sigma_{j}<T\right\}+ \\
& +\sum_{k=0}^{\infty} E\left\{e^{-\alpha \sigma_{k}} R^{(\alpha)}\left(T-\sigma_{k}\right)+e^{-\alpha T} c_{2} ; \sigma_{k}<T \leq \sigma_{k+1}\right\}= \\
& =\sum_{j=0}^{\infty} E\left\{e^{-\alpha \sigma_{j}} R^{(\alpha)}\left(\zeta_{j+1}\right)+e^{-\alpha \sigma_{j+1}} c_{1} ; \sigma_{j+1}<T\right\}+ \\
& +\sum_{k=0}^{\infty} E\left\{e^{-\alpha \sigma_{k}} R^{(\alpha)}\left(T-\sigma_{k}\right)+e^{-\alpha T} c_{2} ; \sigma_{k}<T \leq \sigma_{k+1}\right\} .
\end{aligned}
$$


Desenvolvendo os dois termos da última adição da expressão acima separadamente, temos que, o primeiro termo é dado por

$$
\begin{aligned}
& \sum_{j=0}^{\infty} E\left\{e^{-\alpha \sigma_{j}} R^{(\alpha)}\left(\zeta_{j+1}\right)+e^{-\alpha \sigma j+1} c_{1} ; \sigma_{j+1}<T\right\}= \\
& =\sum_{j=0}^{\infty} E\left\{e^{-\alpha \sigma_{j}} R^{(\alpha)}\left(\zeta_{j+1}\right)+e^{-\alpha \sigma j+1} c_{1} ; \zeta_{j+1}+\sigma_{j}<T\right\}= \\
& =\sum_{j=0}^{\infty} E\left\{e^{-\alpha \sigma_{j}} R^{(\alpha)}\left(\zeta_{j+1}\right)+e^{-\alpha \sigma_{j+1}} c_{1} ; \zeta_{j+1}<T-\sigma_{j}\right\}= \\
& =\sum_{j=0}^{\infty} E\left\{e^{-\alpha \sigma_{j}} R^{(\alpha)}\left(\zeta_{j+1}\right)+e^{-\alpha \sigma j+1} c_{1} ; \zeta_{j+1}<T-\sigma_{j}, \sigma_{j}<T\right\}= \\
& =\sum_{j=0}^{\infty} E\left\{e^{-\alpha \sigma_{j}} E\left\{R^{(\alpha)}\left(\zeta_{j+1}\right)+e^{-\alpha \zeta_{j+1}} c_{1} ; \zeta_{j+1}<T-x \mid \sigma_{j}=x, \sigma_{j}<T\right\}\right\}= \\
& =\sum_{j=0}^{\infty} E\left\{e^{-\alpha \sigma_{j}} E\left\{R^{(\alpha)}(\zeta)+e^{-\alpha \zeta} c_{1} ; \zeta<T-x \mid \sigma_{j}=x, \sigma_{j}<T\right\}\right\}= \\
& =\sum_{j=0}^{\infty} \int_{0}^{T} e^{-\alpha x} E\left\{R^{(\alpha)}(\zeta)+e^{-\alpha \zeta} c_{1} ; \zeta<T-x\right\} d P\left(\sigma_{j} \leq x\right)= \\
& =\int_{0}^{T} e^{-\alpha x} E\left\{R^{(\alpha)}(\zeta)+e^{-\alpha \zeta} c_{l} ; \zeta<T-x\right\} \sum_{j=0}^{\infty} d P\left(\sigma_{j} \leq x\right)= \\
& =\int_{0}^{T} e^{-\alpha x} E\left\{R^{(\alpha)}(\zeta)+e^{-\alpha \zeta} c_{1} ; \zeta<T-x\right\} d U(x) \text {. }
\end{aligned}
$$

O segundo termo pode ser escrito como

$$
\begin{aligned}
& \sum_{j=0}^{\infty} E\left[e^{-\alpha \sigma_{j}} R^{(\alpha)}\left(T-\sigma_{j}\right)+e^{-\alpha T} c_{2} ; \sigma_{j} \leq T<\sigma_{j+1}\right]= \\
& =\sum_{j=0}^{\infty} E\left[e^{-\alpha \sigma_{j}} R^{(\alpha)}\left(T-\sigma_{j}\right)+e^{-\alpha T} c_{2} ; \sigma_{j} \leq T<\zeta_{j+1}+\sigma_{j}\right]= \\
& =\sum_{j=0}^{\infty} E\left[e^{-\alpha \sigma_{j}} R^{(\alpha)}\left(T-\sigma_{j}\right)+e^{-\alpha T} c_{2} ; 0 \leq T-\sigma_{j}<\zeta_{j+1}\right]= \\
& =\sum_{j=0}^{\infty} E\left[e^{-\alpha \sigma_{j}} R^{(\alpha)}\left(T-\sigma_{j}\right)+e^{-\alpha T} c_{2} ; T-\sigma_{j}<\zeta_{j+1}, \sigma_{j}<T\right]=
\end{aligned}
$$




$$
\begin{aligned}
& =\sum_{j=0}^{\infty} E\left[e^{-\alpha \sigma_{j}} E\left[R^{(\alpha)}(T-x)+e^{-\alpha T} c_{2} ; T-x<\left.\zeta_{j+1}\right|_{\sigma_{j}=x}, \sigma_{j}<T\right]\right]= \\
& =\left.\sum_{j=0}^{\infty} \int_{0}^{T} e^{-\alpha x} E\left[R^{(\alpha)}(T-x)+e^{-\alpha T} c_{2} ; T-x<\zeta\right]\right|_{\sigma_{j}=x} d P\left(\sigma_{j} \leq x\right)= \\
& =\int_{0}^{T} e^{-\alpha x} E\left[R^{(\alpha)}(T-x)+e^{-\alpha T} c_{2} ; T-x<\zeta\right] \sum_{j=0}^{\infty} d P\left(\sigma_{j} \leq x\right)= \\
& =\int_{0}^{T} e^{-\alpha x} E\left[R^{(\alpha)}(T-x)+e^{-\alpha T} c_{2} ; T-x<\zeta\right] d U(x) .
\end{aligned}
$$

Combinando-os novamente, concluímos a demonstração.

\section{Seção 2.4- Outras relações de custo.}

Na seção anterior, estabelecemos a relação entre $A^{(\alpha)}(T)$ e $B^{(\alpha)}(T)$. Agora, consideraremos a relação entre $A(T)$ e $A^{(\alpha)}(T)$ e a relação entre $B(T)$ e $B^{(\alpha)}(T)$.

Como na Seção 2.1 assumimos que $R(t)$ é um processo não decrescente e que $c_{1} \mathrm{e}$ $c_{2}$ são não negativos. Em adição, assumiremos que as funções $A(T)$ e $B(T)$ são contínuas à direita e de variação limitada em intervalos compactos.

Teorema 2.4.1: Nas condições acima, temos:

$$
\begin{aligned}
\text { i) } \quad A^{(\alpha)}(T) & =\int_{(0, T]} e^{-\alpha x} d A(x)+E\left[c_{2} e^{-\alpha \delta}\right] \text { onde } \\
\delta=\zeta \wedge T & =\min (\zeta, T) e \\
\text { ii) } \quad B^{(\alpha)}(T) & =\int_{(0, T]} e^{-\alpha x} d B(x)+e^{-\alpha T} c_{2} .
\end{aligned}
$$

Prova: i)

$$
\begin{aligned}
& \int_{(0, T]} e^{-\alpha x} d A(x)=\left.e^{-\alpha x} A(x)\right|_{0} ^{T}+\alpha \int_{0}^{T} e^{-\alpha x} A(x) d x= \\
& =e^{-\alpha T} A(T)-A(0)+\alpha \int_{0}^{T} e^{-\alpha x} E\left[R^{*}(x)\right] d x= \\
& =e^{-\alpha T} A(T)-A(0)+\alpha \int_{0}^{T} e^{-\alpha x}\left\{E\left[R^{*}(x) ; \zeta<T\right]+E\left[R^{*}(x) ; \zeta \geq T\right]\right\} d x=
\end{aligned}
$$




$$
\begin{aligned}
& =e^{-\alpha T}\left(E\left[R^{*}(x) ; \zeta<T\right]+E\left[R^{*}(x) ; \zeta \geq T\right]\right)+ \\
& -c_{2}+E\left\{\alpha \int_{0} e^{-\alpha x}\left(R(x)+c_{2}\right) d x ; \zeta<T\right\}+ \\
& +E\left\{\alpha \int_{\zeta}^{T} e^{-\alpha x}\left[R(\zeta)+c_{1}\right] d x ; \zeta<T\right\}+ \\
& +E\left\{\alpha \int_{0}^{T} e^{-\alpha x}\left[R(x)+c_{2}\right] d x ; \zeta \geq T\right\}= \\
& =e^{-\alpha T}\left(E\left[R(\zeta)+c_{1} ; \zeta<T\right]+E\left[R(T)+c_{2} ; \zeta \geq T\right]\right)-c_{2}+ \\
& +E\left\{\alpha \int_{0}^{\zeta} e^{-\alpha x}\left(R(x)+c_{2}\right) d x ; \zeta<T\right\}+E\left\{\alpha\left[R(\zeta)+c_{1}\right] \int_{\zeta}^{T} e^{-\alpha x} d x ; \zeta<T\right\}+ \\
& +E\left\{\alpha \int_{0}^{T} e^{-\alpha x}\left[R(x)+c_{2}\right] d x ; \zeta \geq T\right\}= \\
& +E\left\{e^{(\alpha)}(T)-E\left\{e^{-\alpha \delta} c_{2}\right\} .\right. \\
& +E\left\{\int_{2}^{(\alpha)}(T)-E\left\{e^{-\alpha \zeta} c_{2} ; \zeta<T\right\}-E\left\{e^{-\alpha T} c_{2} ; \zeta \geq T\right\}=\right. \\
& +E\left\{\alpha \int_{0}^{(\alpha)}(T)+E\left\{c_{2} ; \zeta<T\right\}+E\left\{c_{2} ; \zeta \geq T\right\}-c_{2}+\right. \\
& \left.+e^{-\alpha x} e^{-\alpha}\left(R(x)+c_{2}\right) d x+e^{-\alpha \zeta}\left(R(\zeta)+c_{1}\right) ; \zeta<T\right\}+
\end{aligned}
$$


ii) Queremos provar que

$$
\begin{aligned}
& B^{(\alpha)}(T)=\int_{(0, T]} e^{-\alpha x} d B(x)+e^{-\alpha T} c_{2}= \\
& =\left.e^{-\alpha x} B(x)\right|_{0} ^{T}+\alpha \int_{0}^{T} e^{-\alpha x} B(x) d x+e^{-\alpha T} c_{2}= \\
& =e^{-\alpha T} B(T)-c_{2}+\alpha \int_{0}^{T} e^{-\alpha x} B(x) d x+e^{-\alpha T} c_{2} .
\end{aligned}
$$

Como sabemos que $B(x)=\int_{0}^{x} A(x-y) d U(y)$, será necessário provar que

$$
B^{(\alpha)}(T)=e^{-\alpha T} B(T)-c_{2}+\alpha \int_{0}^{T} e^{-\alpha x}\left(\int_{0}^{x} A(x-y) d U(y)\right) d x+e^{-\alpha T} c_{2} .
$$

Pela parte i) do teorema temos que

$$
A^{(\alpha)}(T-x)=\int_{0}^{(T-x)} e^{-\alpha y} d A(y)+c_{2} E\left[e^{-\alpha\left(\zeta^{\wedge}(T-x)\right)}\right]
$$

$\operatorname{Mas} E\left[e^{-\alpha\left(\zeta^{\wedge}(T-x)\right)}\right]=1-\alpha \int_{0}^{(T-x)} \bar{G}(u) e^{-\alpha u} d u \mathrm{e}$

$$
\begin{aligned}
& A^{(\alpha)}(T-x)=\int_{0}^{(T-x)} e^{-\alpha y} d A(y)= \\
& =\left.e^{-\alpha y} A(y)\right|_{0} ^{(T-x)}+\alpha \int_{0}^{(T-x)} e^{-\alpha y} A(y) d y+E\left[c_{2} e^{-\left(\zeta^{\wedge}(T-x)\right)}\right]= \\
& =e^{-\alpha T-x} A(T-x)-c_{2}+\alpha \int_{0}^{(T-x)} A(y) e^{-\alpha y} d y+c_{2}\left(1-\alpha \int_{0}^{(T-x)} \bar{G}(y) e^{-\alpha y} d y\right) .
\end{aligned}
$$

Substituindo $A^{(\alpha)}(T-x)$ na expressão de $B^{(\alpha)}(T)$, concluímos que 


$$
\begin{aligned}
& B^{(\alpha)}(T)=\int_{0}^{T} \alpha e^{-\alpha x} e^{-\alpha(T-x)} A(T-x) d U(x)+\left[\alpha \int_{0}^{(T-x)} A(y) e^{-\alpha y} d y-c_{2}+\right. \\
& \left.+c_{2}-\alpha c_{2} \int_{0}^{(T-x)} \bar{G}(u) e^{-\alpha y} d y\right] d U(x)=e^{-\alpha T} \int_{0}^{T} A(T-x) d U(x)+ \\
& +\alpha \int_{0}^{T} e^{-\alpha x}\left(\int_{0}^{(T-x)} A(y) e^{-\alpha y} d y\right) d U(x)-\alpha c_{2} \int_{0}^{T}\left(\int_{0}^{(T-x)} \bar{G}(u) e^{-\alpha y} d y\right) d U(x)= \\
& =e^{-\alpha T} B(T)+\alpha \int_{0}^{T} e^{-\alpha x}\left(\int_{x}^{T} A(v-x) e^{-\alpha(v-x)} d v\right) d U(x)+ \\
& -\alpha c_{2} \int_{0}^{T} e^{-\alpha x}\left(\int_{x}^{T} \bar{G}(v-x) e^{-\alpha(v-x)} d v\right) d U(x)= \\
& =e^{-\alpha T} B(T)+\alpha \int_{0}^{T} e^{-\alpha v}\left(\int_{0}^{v} A(v-x) e^{-\alpha x} d U(x)\right) d v+ \\
& -\alpha c_{2} \int_{0}^{T} e^{-\alpha v}\left(\int_{0}^{v} \bar{G}(v-x) d U(x)\right) d v= \\
& =e^{-\alpha T} B(T)+\alpha \int_{0}^{T} e^{-\alpha v}\left(\int_{0}^{x} A(x-y) e^{-\alpha y} d U(y)\right) d x+ \\
& -\alpha c_{2} \int_{0}^{T} e^{-\alpha v}\left(\int_{0}^{v}(1-G(v-x)) d U(x)\right) d v .
\end{aligned}
$$

Desenvolvendo o último termo da expressão acima, obtemos

$$
\begin{aligned}
& \alpha c_{2} \int_{0}^{T}\left(\int_{0}^{v}(1-G(v-x)) d U(x)\right) e^{-\alpha v} d v=\alpha c_{2} \int_{0}^{T}\left(U(v)-\int_{0}^{v}(G(v-x)) d U(x)\right) e^{-\alpha v} d v= \\
& =\alpha c_{2} \int_{0}^{T}(U(v)-V(v)) e^{-\alpha v} d v=\alpha c_{2} \int_{0}^{T} e^{-\alpha v} d v=c_{2}\left(1-e^{-\alpha T}\right)
\end{aligned}
$$


Cap. 2 -Relação entre os custos esperados com desconto nas pol. de man. por idade e por bloco

Então

$$
\begin{aligned}
& B^{(\alpha)}(T)=e^{-\alpha T} B(T)+\alpha \int_{0}^{T} e^{-\alpha v}\left(\int_{0}^{x} A(x-y) e^{-\alpha y} d U(y)\right) d x+ \\
& -\alpha c_{2} \int_{0}^{T} e^{-\alpha v}\left(\int_{0}^{v}(1-G(v-x)) d U(x)\right) d v= \\
& =e^{-\alpha T} B(T)+\alpha \int_{0}^{T} e^{-\alpha v}\left(\int_{0}^{x} A(x-y) e^{-\alpha y} d U(y)\right) d x-c_{2}\left(1-e^{-\alpha T}\right) .
\end{aligned}
$$

Relacionamento entre os custos das politicas de manutenção por idade e por bloco - pág. 45 
Capítulo 3

Políticas de manutenção ótimas por idade e por bloco para um modelo geral 


\section{Seção 3.1 - A função de custo marginal}

No Capítulo 1, calculamos o custo esperado por unidade de tempo, no decorrer do tempo para as políticas de manutenção por idade e por bloco e denotamos por $J_{A}(T)$ e $J_{B}(T)$, respectivamente. No Capítulo 2, onde consideramos um desconto exponencial de $\alpha>0$, calculamos a esperança do custo total com desconto, denotada por $J^{(\alpha)}{ }_{A}(T)$ no caso de uma política de manutenção por idade e por $J^{(\alpha)}{ }_{B}(T)$ no caso de uma política de manutenção por bloco.

Demonstramos a validade das expressões

$$
\begin{aligned}
& J_{A}(T)=\frac{A(T)}{E[\delta]}=\frac{A(T)}{\int_{0}^{T} \bar{G}(u) d u}, J_{B}(T)=\frac{B(T)}{T}, \\
& J_{A}^{(\alpha)}(T)=\frac{A^{(\alpha)}(T)}{1-E\left[e^{-\alpha(\delta)}\right]} e J^{(\alpha)}(T)=\frac{B^{(\alpha)}(T)}{1-e^{-\alpha T}}
\end{aligned}
$$

onde

$$
\begin{aligned}
& A(T)=E\left[R(\zeta)+c_{1} ; \zeta<T\right]+E\left[R(T)+c_{2} ; \zeta \geq T\right] \\
& B(T)=\int_{[0, T)} A(T-x) d U(x), \\
& A^{(\alpha)}(T)=\int_{[0, T)} e^{-\alpha x} d A(x)+c_{2} E\left[e^{-\alpha(\zeta \wedge T)}\right] \mathrm{e} \\
& B^{(\alpha)}(T)=\int_{[0, T)} e^{-\alpha x} d B(x)+c_{2} e^{-\alpha T} .
\end{aligned}
$$

As expressões acima dependem do parâmetro de manutenção $T$ e, no que segue, serão chamadas funções objetivas.

Neste capítulo, usando resultados de Chen e Savits (1.989), estamos interessados em minimizar estas funções objetivas com respeito ao parâmetro $T$. 
Notemos que em todos os casos a função objetiva tem a forma $J(T)=\frac{N(T)}{D(T)}$.

Os pontos críticos dessa função são obtidos igualando a derivada desta expressão a zero, isto é,

$$
\begin{aligned}
& \frac{d(J(T))}{d T}=\frac{N^{\prime}(T) D(T)-D^{\prime}(T) N(T)}{D^{2}(T)}=0, \text { então } \\
& N^{\prime}(T) D(T)-D^{\prime}(T) N(T)=0, \text { ou seja, } \\
& N^{\prime}(T) D(T)=D^{\prime}(T) N(T) \text { e } \\
& \frac{N^{\prime}(T)}{D^{\prime}(T)}=\frac{N(T)}{D(T)}=J(T) .
\end{aligned}
$$

Assim, se existir, deveremos obter o $T$ ótimo quando $\eta(T)=J(T)$.

Definição 3.1.1: Se $\mathrm{N}$ e $\mathrm{D}$ são continuamente diferenciáveis em $[0, \infty)$ com $D^{\prime}(T)>0$ e $T \in[0, \infty)$, definimos a função de custo marginal da função objetiva $J(T)$ por

$$
\eta(T)=\frac{N^{\prime}(T)}{D^{\prime}(T)}
$$

Para desenvolvermos as expressões das funções de custo marginal, assumiremos que existe uma função mensurável $\phi(u, y)$ tal que

$$
E[R(x) \mid \zeta=y]=\int_{0}^{x} \phi(u, y) d u \text { para todo } 0 \leq x \leq y \text {. As expressões para } \eta(T) \text { são }
$$

dadas pelo teorema seguinte:

Teorema 3.1.2 : Seja uma função mensurável $\phi(u, y)$ tal que $E[R(x) \mid \zeta=y]=\int_{0}^{\infty} \phi(u, y) d u$. Suponhamos que a função de distribuição $G$ de $\zeta$ tenha função de densidade $g$ e que 


$$
\begin{aligned}
& \text { i) } \eta_{A}(T)=\frac{1}{\bar{G}(T)} \int_{T}^{\infty} \phi(T, y) g(y) d y+\left(c_{1}-c_{2}\right) r_{G}(T), \\
& \text { ii) } \eta_{B}(T)=\int[0, T)\left\{\eta_{A}(T-x) \bar{G}(T-x)+c_{2} g(T-x)\right\} d U(x), \\
& \text { iii) }{ }^{(\alpha)}{ }_{A}(T)=\frac{1}{\alpha} \eta_{A}(T),-c_{2} \text { e } \\
& \text { iv) }{ }^{(\alpha)}{ }_{B}(T)=\frac{1}{\alpha} \eta_{B}(T)-c_{2} .
\end{aligned}
$$

\section{Prova:}

i) Como $J_{A}(T)=\frac{A(T)}{\int_{0}^{T} \bar{G}(u) d u}=\frac{N(T)}{D(T)}$, temos $\eta_{A}(T)=\frac{N^{\prime}(T)}{D^{\prime}(T)}=\frac{A^{\prime}(T)}{\bar{G}(T)}$.

Contudo,

$$
\begin{aligned}
& A(T)=E\left[R(\zeta)+c_{1} ; \zeta<T\right]+E\left[R(T)+c_{2} ; \zeta \geq T\right]= \\
& =E\left\{E\left[R(\zeta)+c_{1} ; \zeta<T\right] \zeta\right\}+E\left\{E\left[R(T)+c_{2} ; \zeta \geq T\right] \zeta\right\}= \\
& =\int_{0}^{T} E\left[R(\zeta)+c_{1} ; \zeta=y\right] g(y) d y+\int_{T}^{\infty} E\left[R(T)+c_{2} ; \zeta=y\right] g(y) d y= \\
& =\int_{0}^{T} \int_{0}^{y} \phi(u, y) d u g(y) d y+c_{1} G(T)+\int_{T}^{\infty} \int_{0}^{T} \phi(u, y) d u g(y) d y+c_{2} \bar{G}(T)= \\
& =\left(c_{1}-c_{2}\right) G(T)+c_{2}+\int_{0}^{T} \int_{u} g(y) \phi(u, y) d y d u+\int_{0}^{T} \int_{T}^{\infty} g(y) \phi(u, y) d y d u= \\
& =\left(c_{1}-c_{2}\right) G(T)+c_{2}+\int_{0}^{T} \int_{u}^{\infty} g(y) \phi(u, y) d y d u .
\end{aligned}
$$

Se $\int_{0}^{u} \phi(u, y) g(y) d y$ é contínua em $u$, então $A(T)$ é continuamente diferenciável e 


$$
A^{\prime}(T)=\int_{T}^{\infty} \phi(T, y) g(y) d y+\left(c_{1}-c_{2}\right) g(T) \text {. }
$$

Assim,

$$
\begin{aligned}
& \eta_{A}(T)=\frac{A^{\prime}(T)}{\bar{G}(T)}=\frac{1}{\bar{G}(T)}\left\{\int_{T}^{\infty} \phi(T, y) g(y) d y+\left(c_{1}-c_{2}\right) g(T)\right\}= \\
& =\frac{\int_{T}^{\infty} \phi(T, y) g(y) d y}{\bar{G}(T)}+\left(c_{1}-c_{2}\right) \frac{g(T)}{\bar{G}(T)}=\frac{\int_{T}^{\infty} \phi(T, y) g(y) d y}{\bar{G}(T)}+\left(c_{1}-c_{2}\right) r_{G}(T) .
\end{aligned}
$$

ii)Como $J_{B}=\frac{B(T)}{T}=\frac{N(T)}{D(T)}$, temos $\eta_{B}(T)=B^{\prime}(T)$.

Se $B(T)=\int_{[0, T)} A(T-x) d U(x)$ e $A$ é continuamente diferenciável, podemos escrever

$$
\begin{aligned}
B(T) & =\int_{[0, T)}\left[\int_{x}^{T} A^{\prime}(y-x) d y+A(0)\right] d U(x)= \\
& =\int_{0}^{T}\left[\int_{0}^{y} A^{\prime}(y-x) d U(x)\right] d y+c_{2} U(T) .
\end{aligned}
$$

Como $G$ tem função de densidade $g$, podemos afirmar que $V(T)=U(T)-1$ tem função de densidade $v_{G}$ e portanto

$$
\begin{aligned}
\eta_{B}(T) & =B^{\prime}(T)=\int_{0}^{T} A^{\prime}(T-x) d U(x)+c_{2} v_{G}(T)= \\
& =\int_{0}^{T}\left\{\eta_{A}(T-x) \bar{G}(T-x)+c_{2} g(T-x)\right\} d U(x) .
\end{aligned}
$$

iii)Neste caso, $J_{A}^{(\alpha)}=\frac{A^{(\alpha)}(T)}{1-E\left\{e^{-\alpha \delta}\right\}}=\frac{A^{(\alpha)}(T)}{\alpha \int_{0}^{T} \bar{G}(u) e^{-\alpha u} d u} e$ 


$$
\begin{aligned}
A^{(\alpha)}(T) & =\int_{[0, T)} e^{-\alpha u} d A(u)+c_{2} E\left\{e^{-\alpha \delta}\right\}= \\
& =e^{-\alpha T} A(T)-A(0)+\alpha \int_{[0, T)} e^{-\alpha u} A(u) d u+c_{2} E\left\{e^{-\alpha \delta}\right\}= \\
& =e^{-\alpha T} A(T)-A(0)+\alpha \int_{[0, T)} e^{-\alpha u} A(u) d u+c_{2}\left\{1-\alpha \int_{0}^{T} \bar{G}(u) e^{-\alpha u} d u\right\} .
\end{aligned}
$$

Então

$$
\begin{aligned}
& \begin{aligned}
\frac{d^{\prime}\left[A^{(\alpha)}(T)\right]}{d T} & =-\alpha e^{-\alpha T} A(T)+e^{-\alpha T} A^{\prime}(T)+\alpha e^{-\alpha T} A(T)-\alpha c_{2} e^{-\alpha T} \bar{G}(T)= \\
& =e^{-\alpha T} A^{\prime}(T)-\alpha c_{2} e^{-\alpha T} \bar{G}(T) \mathrm{e}
\end{aligned} \\
& \eta_{A}^{(\alpha)}(T)=\frac{N^{\prime}(T)}{D^{\prime}(T)}=\frac{e^{-\alpha T} A^{\prime}(T)-\alpha c_{2} e^{-\alpha T} \bar{G}(T)}{\alpha e^{-\alpha T} \bar{G}(T)}=\frac{A^{\prime}(T)}{\alpha \bar{G}(T)}-c_{2}=\frac{\eta_{A}(T)}{\alpha}-c_{2} .
\end{aligned}
$$

iv)Como $J_{B}^{(\alpha)}=\frac{B^{(\alpha)}(T)}{1-e^{-\alpha T}} \mathrm{e}$

$$
\begin{aligned}
B^{(\alpha)}(T) & =\int_{[0, T)} e^{-\alpha x} d B(x)+e^{-\alpha T} c_{2}= \\
& =e^{-\alpha T} B(T)-B(0)+\alpha \int_{[0, T)} e^{-\alpha x} B(x) d x+e^{-\alpha T} c_{2}, \text { temos }
\end{aligned}
$$

$\frac{d B^{(\alpha)}(T)}{d T}=-\alpha e^{-\alpha T} B(T)+e^{-\alpha T} B^{\prime}(T)+\alpha e^{-\alpha T} B(T)-\alpha e^{-\alpha T} c_{2}$.

Assim,

$$
\eta_{B}^{(\alpha)}(T)=\frac{e^{-\alpha T}\left(B^{\prime}(T)-\alpha c_{2}\right)}{\alpha e^{-\alpha T}}=\frac{\eta_{B}(T)}{\alpha}-c_{2} .
$$




\section{Observações:}

i)Uma condição suficiente para $\int_{T}^{\infty} \phi(T, y) g(y) d y$ ser contínua em $T$ é que $\phi(u, y)$ seja contínua em $u$ para cada $y$ e, para cada $u$, seja limitada por uma função $h$ integrável em uma vizinhança de $u$.

ii)Quando $\phi(u, y)$ independe de $y$, isto é, $\phi(u, y)=\Phi(u)$, a expressão para a função de custo marginal de uma política de manutenção por idade pode ser simplificada por

$$
\begin{aligned}
\eta_{A}(T) & =\frac{1}{\bar{G}(T)} \int_{T}^{\infty} \phi(T, y) g(y) d y+\left(c_{1}-c_{2}\right) r_{G}(T)= \\
& =\frac{1}{\bar{G}(T)} \Phi(T) \int_{T}^{\infty} g(y) d y+\left(c_{1}-c_{2}\right) r_{G}(T)= \\
& =\Phi(T)+\left(c_{1}-c_{2}\right) r_{G}(T) .
\end{aligned}
$$

iii)Note que a expressão para $\eta_{B}(T)$ não depende de $c_{2}$ pois

$$
\begin{aligned}
& \eta_{A}(T) \bar{G}(T)+c_{2} g(T)=\int_{T}^{\infty} \phi(T, y) g(y) d y+c_{1} g(T) \mathrm{e} \\
& \eta_{\mathrm{B}}(T)=\int_{[0, T)}\left\{\eta_{A R}(T-x) \bar{G}(T-x)+c_{1} g(T-x)\right\} d U(x) \text { onde } \\
& \eta_{A R}(T)=\frac{1}{\bar{G}(T)} \int_{T}^{\infty} \phi(T, y) g(y) d y .
\end{aligned}
$$




\section{Seção 3.2. - Resultados de Otimização}

Nesta seção consideraremos o problema de otimização das funções objetivas da forma $J(T)$, isto é, minimizar estas expressões com relação ao parâmetro de manutenção $T$. Este problema de minimização foi estudado por Pury e Singh (1.985) neste teorema:

Teorema 3.2.1: Seja a função objetiva $J(T)=\frac{N(T)}{D(T)}$ com $N$ e $D$ continuamente diferenciáveis em $[0, \infty), N(0)>0, D(0)=0$ e $D^{\prime}(T)>0$ para todo $T \geq 0$. Seja $\eta(T)=\frac{N^{\prime}(T)}{D^{\prime}(T)}$ a função de custo marginal associada a $J(T)$. Se $\eta(T)$ é uma função não decrescente em $[0, \infty)$ então

i) $\eta(T) D(T)-N(T)$ é não decrescente em $[0, \infty$ ) e portanto tem um limite $L$ (que pode ser $+\infty$ quando $T \rightarrow \infty$ ).

ii)Se $\mathrm{L}>0$, existe um valor mínimo $T_{0}, 0<T_{0}<\infty$, onde $J(T)$ assume seu mínimo global e $\eta\left(T_{0}\right)=J\left(T_{0}\right)$.

iii)Qualquer solução finita da equação $\eta(T)=J(T)$ é um ponto de mínimo global para $J(T)$.

iv)Se $\eta$ é estritamente crescente, existe no máximo uma solução para a equação $\eta(T)=J(T)$.

v)Se $L=0$, então $J(T)$ é uma função não crescente, entretanto $J(T)$ pode assumir um valor mínimo em $\left[T_{0}, \infty\right)$, para algum valor finito $T_{0}$ 


\section{Prova:}

i)Seja $0 \leq t_{1}<t_{2}$. Temos

$$
\begin{aligned}
& \left(\eta\left(t_{2}\right) D\left(t_{2}\right)-N\left(t_{2}\right)\right)-\left(\eta\left(t_{1}\right) D\left(t_{1}\right)-N\left(t_{1}\right)\right)= \\
& =\eta\left(t_{2}\right) D\left(t_{2}\right)-N\left(t_{2}\right)-\eta\left(t_{1}\right) D\left(t_{1}\right)+N\left(t_{1}\right)+\eta\left(t_{2}\right) D\left(t_{1}\right)-\eta\left(t_{2}\right) D\left(t_{1}\right)= \\
& =\left[\eta\left(t_{2}\right)-\eta\left(t_{1}\right)\right] D\left(t_{1}\right)+\eta\left(t_{2}\right)\left[D\left(t_{2}\right)-D\left(t_{1}\right)\right]-N\left(t_{2}\right)+N\left(t_{1}\right)= \\
& =\left[\eta\left(t_{2}\right)-\eta\left(t_{1}\right)\right] D\left(t_{1}\right)+\eta\left(t_{2}\right) \int_{t_{1}}^{t_{2}} \eta\left(t_{2}\right) D^{\prime}(t) d t-\int_{t_{1}}^{t_{2}} N^{\prime}(t) d t= \\
& =\left[\eta\left(t_{2}\right)-\eta\left(t_{1}\right)\right] D\left(t_{1}\right)+\int_{t_{1}}^{t_{2}}\left(\eta\left(t_{2}\right) D^{\prime}(t)-N^{\prime}(t)\right) d t= \\
& =\left[\eta\left(t_{2}\right)-\eta\left(t_{1}\right)\right] D\left(t_{1}\right)+\int_{t_{1}}^{t_{2}}\left(\eta\left(t_{2}\right) D^{\prime}(t)-\eta(t) D^{\prime}(t)\right) d t= \\
& =\left[\eta\left(t_{2}\right)-\eta\left(t_{1}\right)\right] D\left(t_{1}\right)+\int_{t_{1}}^{t_{2}}\left(\eta\left(t_{2}\right)-\eta(t)\right) D^{\prime}(t) d t .
\end{aligned}
$$

Observemos que $\left[\eta\left(t_{2}\right)-\eta\left(t_{1}\right)\right] D\left(t_{1}\right) \geq 0$ pois $\eta(t)$ é não-decrescente em $t$ e $D(t)>0$ uma vez que $D(t)$ é não-decrescente para $t>0$ e $D(0)=0$. Como $D^{\prime}(t)>0$ para $t>0$ concluímos também que $\int_{t_{1}}^{t_{2}}\left(\eta\left(t_{2}\right)-\eta(t)\right) D^{\prime}(t) d t \geq 0$.

$$
\text { Portanton }\left(t_{2}\right) D\left(t_{2}\right)-N\left(t_{2}\right)-\left(\eta\left(t_{1}\right) D\left(t_{1}\right)-N\left(t_{1}\right)\right) \geq 0 \text { e } \eta(t) D(t)-N(t) \text { é não- }
$$
decrescente em $t>0$.

ii)A expressão $J(T)$ assume o seu ponto crítico quando $\eta(T) D(T)-N(T)=0$. Como $\eta(0) D(0)-N(0)<0, \eta(T) D(T)-N(T)$ é não decrescente por i) e $\lim _{t \rightarrow \infty} \eta(t) D(t)-N(t)=L>0$ por hipótese, podemos afirmar que existe um ponto $T_{0}$ onde $\eta\left(T_{0}\right) D\left(T_{0}\right)-N\left(T_{0}\right)=0$, isto é, que é crítico para $J(T)$. Mostraremos que $T_{0}$ é um ponto de mínimo global de $J(T)$. 
Para $0<T<T_{0}$, temos

$$
\begin{aligned}
& J(T)-J\left(T_{0}\right)=\frac{N(T)}{D(T)}-\frac{N\left(T_{0}\right)}{D\left(T_{0}\right)}=\frac{N(T) D\left(T_{0}\right)-N\left(T_{0}\right) D(T)}{D(T) D\left(T_{0}\right)}= \\
& =\frac{N(T) D\left(T_{0}\right)-\eta\left(T_{0}\right) D\left(T_{0}\right) D(T)}{D(T) D\left(T_{0}\right)}=\frac{N(T)-\eta\left(T_{0}\right) D(T)}{D(T)}= \\
& =\frac{N(T)-\eta\left(T_{0}\right) D(T)+\eta\left(T_{0}\right) D\left(T_{0}\right)-\eta\left(T_{0}\right) D\left(T_{0}\right)}{D(T)}= \\
& =\frac{\eta\left(T_{0}\right)\left[D\left(T_{0}\right)-D(T)\right]-\eta\left(T_{0}\right) D\left(T_{0}\right)+N(T)}{D(T)}= \\
& =\frac{\eta\left(T_{0}\right)\left[D\left(T_{0}\right)-D(T)\right]-\left[N\left(T_{0}\right)-N(T)\right]}{D(T)}= \\
& =\frac{\eta\left(T_{0}\right) \int_{T}^{T_{0}} D^{\prime}(t) d t-\int_{T}^{T_{0}} N^{\prime}(t) d t}{D(T)}=\frac{\eta\left(T_{0}\right) \int_{T}^{T_{0}} D^{\prime}(t) d t-\int_{T}^{T_{0}} \eta(t) D^{\prime}(t) d t}{D(T)}= \\
& =\frac{\int_{T}^{T_{0}}\left[\eta\left(T_{0}\right)-\eta(t)\right] D^{\prime}(t) d t}{D(T)}>0 \text {, pois } \eta(t) \mathrm{e}^{\prime} \text { não decrescente e } D^{\prime}(t)>0 \text {. }
\end{aligned}
$$

Para $0<T_{0}<T$,

$$
\begin{aligned}
& J(T)-J\left(T_{0}\right)=\frac{\eta\left(T_{0}\right)\left[D\left(T_{0}\right)-D(T)\right]-\left[N\left(T_{0}\right)-N(T)\right]}{D(T)}= \\
& =\frac{\int_{T_{0}}^{T} N^{\prime}(t) d t-\eta\left(T_{0}\right) \int_{T_{0}}^{T} D^{\prime}(t) d t}{D(T)}=\frac{\int_{T}^{T_{0}} \eta(t) D^{\prime}(t) d t-\eta\left(T_{0}\right) \int_{T}^{T_{0}} D^{\prime}(t) d t}{D(T)}= \\
& =\frac{\int_{T_{0}}^{T}\left[\eta(t)-\eta\left(T_{0}\right)\right] D^{\prime}(t) d t}{D(T)} .
\end{aligned}
$$


Assim, temos novamente que $J(T)-J\left(T_{0}\right)>0$, pois $\eta(t) \mathrm{e}^{\prime}$ não decrescente, $D^{\prime}(t)>0$ e provamos que $T_{0}$ é um ponto de mínimo global de $J(T)$. Temos também que em $T_{0}, \eta\left(T_{0}\right) D\left(T_{0}\right)-N\left(T_{0}\right)=0$ e portanto $\eta\left(T_{0}\right)=\frac{N\left(T_{0}\right)}{D\left(T_{0}\right)}=J\left(T_{0}\right)$.

iii)Podemos mostrar que qualquer solução finita de $\eta(T)=J(T)$ é um ponto de mínimo global de $J(T)$ do mesmo modo que em ii).

iv)Como $\eta(T)$ é estritamente crescente, existe um único ponto tal que $\eta(T)=J(T)$, isto é, uma única solução para esta equação.

v)Como $\lim _{t \rightarrow \infty} \eta(t) D(t)-N(t)=0$ e por i) $\eta(t) D(t)-N(t)$ é não decrescente, concluímos que $\eta(t) D(t)-N(t) \leq 0$.

Então

$$
\begin{aligned}
& \frac{d(J(T))}{d T}=\frac{N^{\prime}(T) D(T)-D^{\prime}(T) N(T)}{D^{2}(T)}=\frac{\eta(T) D^{\prime}(T) D(T)-D^{\prime}(T) N(T)}{D^{2}(T)}= \\
& =\frac{D^{\prime}(T)[\eta(T) D(T)-N(T)]}{D^{2}(T)}<0 \text { pois } D^{\prime}(T)>0 \text { e }[\eta(T) D(T)-N(T)] \leq 0 .
\end{aligned}
$$

Portanto $J(T)$ é não crescente. Entretanto o limite pode ser alcançado para algum

$T_{0}$ finito.

Corolário 3.2.2: Nas condições do Teorema 3.2.1, se as funções $N$ e $D$ são limitadas, temos que

$L>0$ se e somente se $\eta(\infty)=\lim _{T \rightarrow \infty} \eta(T)>\lim _{T \rightarrow \infty} J(T)=J(\infty)$

Prova: Basta notar que

$$
\lim _{T \rightarrow \infty} \eta(T) D(T)-N(T)>0 \Leftrightarrow \lim _{T \rightarrow \infty} \eta(T)-J(T)>0 \Leftrightarrow \lim _{T \rightarrow \infty} \eta(T)>\lim _{T \rightarrow \infty} J(T) .
$$




\section{Seção 3.3 - Exemplos}

Nos exemplos a seguir, aplicaremos o Teorema 3.2.1 em cada uma das funções objetivas para determinar $R, \zeta, c_{1}$ e $c_{2}$ de maneira que a função de custo marginal associada $\eta$ seja não decrescente e o limite $L$ seja positivo.

\section{Exemplo 3.3.1 - Política de manutenção por idade sem desconto}

Teorema 3.3.1.1: Seja $\phi(u, y)$ não decrescente em $(u, y) \operatorname{com} \phi(T, y) \rightarrow c_{R}$ uniformemente em uma vizinhança de $y=\infty$ quando $T \rightarrow \infty$. Assumamos que $\zeta$ é IFR e $c_{1} \geq c_{2}$. Então se

$$
\begin{aligned}
& c_{R}+\left(c_{1}-c_{2}\right) r_{G}(\infty)>\frac{\left(E[R(\zeta)]+c_{1}\right)}{\mu_{1}} \text {, onde } \mu_{1}=E[\zeta], \quad \text { existe um } \\
& T_{0}, 0<T_{0}<\infty \text {, que minimiza } J_{A}(T) .
\end{aligned}
$$

Prova: Notemos que

$$
\begin{aligned}
J_{A}(T)= & \frac{A(T)}{T} \operatorname{com} A(T)=E\left[R(\zeta)+c_{1} ; \zeta<T\right]+E\left[R(T)+c_{2} ; \zeta \geq T\right] \text { e que } \\
& \int_{0} \bar{G}(u) d u \\
\eta_{A}(T)= & \frac{1}{\bar{G}(T)} \int_{T}^{\infty} \phi(T, y) g(y) d y+\left(c_{1}-c_{2}\right) r_{G}(T) .
\end{aligned}
$$

Não é difícil mostrar que o primeiro termo de $\eta_{A}$ é não decrescente em $T$ se $\phi(\mathrm{u}, \mathrm{y})$ é não decrescente em $u$ e $\cdot y$. Como por hipótese $c_{1} \geq c_{2}$ e $r_{G}$ é não decrescente $(\zeta$ é IFR), $\eta_{A}$ é não decrescente. Como

$$
\begin{aligned}
& A(T)=E\left[R(\zeta)+c_{1} ; \zeta<T\right]+E\left[R(T)+c_{2} ; \zeta \geq T\right]= \\
& =E[R(\zeta) ; \zeta<T]+c_{1} P\{\zeta<T\}+E[R(T) ; \zeta \geq T]+c_{2} P\{\zeta \geq T\} \leq \\
& \leq E[R(\zeta) ; \zeta<T]+c_{1}+c_{2}+E[R(T) ; \zeta \geq T] \leq \\
& \leq E[R(\zeta) ; \zeta<T]+c_{1}+c_{2}+E[R(\zeta) ; \zeta \geq T]= \\
& =E[R(\zeta)]+c_{1}+c_{2} \mathrm{e}
\end{aligned}
$$




$$
\int_{0}^{T} \bar{G}(u) d u \leq \int_{0}^{\infty} \bar{G}(u) d u=E[\zeta]=\mu_{1}
$$

concluímos que o numerador e o denominador da expressão de $J_{A}(T)$ são limitados e podemos então aplicar o Corolário 3.2.2.

Segue do Corolário 3.2.2 que $L>0$ se e somente se $\eta_{A}(\infty)>J_{A}(\infty)=\frac{\left(E[R(\zeta)]+c_{1}\right)}{\mu_{1}}$. Como por hipótese $\phi(T, y) \rightarrow c_{R}$ uniformemente em uma vizinhança de $y=\infty$ quando $T \rightarrow \infty$ temos que $\eta_{A}(\infty)=\lim _{T \rightarrow \infty} \eta_{A}(T)=c_{R}+\left(c_{1}-c_{2}\right) r_{G}(\infty)$ e a condição acima é satisfeita se

$$
\eta_{A}(\infty)=c_{R}+\left(c_{1}-c_{2}\right) r_{G}(\infty)>J_{A}(\infty)=\frac{\left(E[R(\zeta)]+c_{1}\right)}{\mu_{1}}
$$

\section{Exemplo 3.3.2 - Política de manutenção por idade com desconto}

Teorema 3.3.2.1: Seja $\phi(u, y)$ não decrescente em $(u, y) \operatorname{com} \phi(T, y) \rightarrow c_{R}$ uniformemente em uma vizinhança de $y=\infty$ quando $T \rightarrow \infty$ e assumamos que $\zeta$ é IFR e $c_{1} \geq c_{2}$. Então se

$$
\left\{\frac{1}{\alpha}\left[c_{R}+\left(c_{1}-c_{2}\right) r_{G}(\infty)\right]-c_{2}\right\}>\left\{E\left[\frac{\alpha \int_{0}^{\infty} e^{-\alpha v} R(v \wedge \zeta) d v+c_{1} E\left[e^{-\alpha \zeta}\right]}{\left\{1-E\left[e^{-\alpha \zeta}\right]\right\}}\right]\right\},
$$

existe $T_{0}, 0<T_{0}<\infty$, que minimiza $J^{(\alpha)}(T)$.

Prova: De acordo com o Teoremas 2.1.1 e o Teorema 3.1.2 iii),

$$
\begin{aligned}
& J_{A}^{(\alpha)}(T)=\frac{A^{(\alpha)}(T)}{\left\{1-E\left[e^{-\alpha \delta}\right]\right\}} \operatorname{com} A^{(\alpha)}(T)=\int_{[0, T)} e^{-\alpha x} d A(x)+c_{2} E\left[e^{-\alpha(\zeta \wedge T)}\right] \mathrm{e} \\
& \eta_{A}{ }^{(\alpha)}(T)=\frac{1}{\alpha} \eta_{A}(T)-c_{2} .
\end{aligned}
$$


$\eta_{A}{ }^{(\alpha)}$ é não decrescente pois, pelo exemplo anterior, $\eta_{A}$ é não decrescente.

Como o numerador e o denominador da expressão de $J_{A}^{(\alpha)}(T)$ são limitados, podemos utilizar o Corolário 3.2 .2 e concluir que $L>0$ se e somente se $\eta_{A}^{(\alpha)}(\infty)>J_{A}^{(\alpha)}(\infty)$. Pelo exemplo anterior,

$$
\eta_{A}^{(a)}(\infty)=\frac{1}{\alpha} \eta_{A}(\infty)-c_{2}=\left\{\frac{1}{\alpha}\left[c_{R}+\left(c_{1}-c_{2}\right) r_{G}(\infty)\right]-c_{2}\right\} .
$$

Além disso,

$$
J_{A}^{(\alpha)}(\infty)=\frac{A^{(\alpha)}(\infty)}{1-E\left[e^{-\alpha \zeta}\right]}
$$

Para o cálculo de $A^{(\alpha)}(\infty)$ utilizaremos a expressão de $A^{(\alpha)}(T)$ obtida no Lema 2.3.2:

$$
\begin{aligned}
& A^{(\alpha)}(T)=E\left[\alpha \int_{0}^{\zeta} e^{-\alpha v} R(v+) d v+e^{-\alpha \zeta}\left\{R(\zeta)+c_{1}\right\} ; \zeta<T\right]+ \\
& +E\left[\alpha \int_{0}^{T} e^{-\alpha v} R(v+) d v+e^{-\alpha T}\left\{R(T)+c_{1}\right\} ; \zeta \geq T\right]
\end{aligned}
$$

Assim,

$$
\begin{aligned}
A^{(\alpha)}(\infty) & =E\left[\alpha \int_{0}^{\zeta} e^{-\alpha v} R(v+) d v+e^{-\alpha \zeta}\left\{R(\zeta)+c_{1}\right\}\right]= \\
& =E\left[\alpha \int_{0}^{\zeta} e^{-\alpha v} R(v+) d v+e^{-\alpha \zeta} c_{1}+\alpha \int_{\zeta}^{\infty} e^{-\alpha v} R(\zeta) d v\right]= \\
& =E\left[\alpha \int_{0}^{\infty} e^{-\alpha v} R(v \wedge \zeta) d v+e^{-\alpha \zeta} c_{1}\right] \mathrm{e}
\end{aligned}
$$




$$
J_{A}^{(\alpha)}(\infty)=\frac{E\left[\alpha \int_{0}^{\infty} e^{-\alpha v} R(v \wedge \zeta) d v+e^{-\alpha \zeta} c_{1}\right]}{1-E\left[e^{-\alpha \zeta}\right]} .
$$

As condições do Corolário 3.2.2 estarão satisfeitas se

$$
\begin{aligned}
& \eta_{A}{ }^{(\alpha)}(\infty)=\frac{1}{\alpha} \eta_{A}(\infty)-c_{2}=\left\{\frac{1}{\alpha}\left[c_{R}+\left(c_{1}-c_{2}\right) r_{G}(\infty)\right]-c_{2}\right\}> \\
& >\frac{E\left[\alpha \int_{0}^{\infty} e^{-\alpha v} R(v \wedge \zeta) d v+e^{-\alpha \zeta} c_{1}\right]}{1-E\left[e^{-\alpha \zeta}\right]}=J_{A}^{(\alpha)}(\infty),
\end{aligned}
$$

o que demonstra o teorema.

\section{Exemplo 3.3.3 - Política de manutenção por bloco sem desconto}

Neste caso, temos $J_{B}(T)=\frac{B(T)}{T}$ com $B(T)=\int_{[0, T)} A(T-x) d U(x)$ e pela observação iii) do Teorema 3.1.2,

$$
\eta_{\mathrm{B}}(T)=\int_{[0, T)}\left\{\eta_{A R}(T-x) \bar{G}(T-x)+c_{1} g(T-x)\right\} d U(x) .
$$

Uma condição necessária para que $\eta_{B}$ seja não decrescente é que o integrando $\left\{\eta_{A R}(T) \bar{G}(T)+c_{1} g(T)\right\}$ seja não decrescente. Mas

$$
\begin{aligned}
& \int_{0}^{\infty}\left\{\eta_{A R}(t) \bar{G}(t)+c_{1} g(t)\right\} d t=\int_{0}^{\infty}\left\{\frac{1}{\bar{G}(t)}\left[\int_{t}^{\infty} \phi(t, y) g y d y\right] \bar{G}(t)+c_{1} g(t)\right\} d t= \\
& =\int_{0}^{\infty} \int_{t}^{\infty} \phi(t, y) g(y) d y d t+\int_{0}^{\infty} c_{1} g(t) d t=\int_{0}^{\infty} \int_{0}^{y} \phi(t, y) d t g(y) d y+\int_{0}^{\infty} c_{1} g(t) d t= \\
& =\int_{0}^{\infty} E[R(y) / \zeta=y] g y d y+c_{1} \int_{0}^{\infty} g(t) d t=E[R(\zeta)]+c_{1}<\infty
\end{aligned}
$$

e o integrando é decrescente. Contudo esta condição não é suficiente e adotaremos, por hipótese que $\eta_{B}$ seja não decrescente. 
Como o denominador da expressão de $J_{B}(T)$ não é limitado, não podemos utilizar o Corolário 3.2.2. Calcularemos o limite $L_{B}=\lim _{T \rightarrow \infty}\left[T \eta_{B}(T)-B(T)\right]$ utilizando transformadas de Laplace.

Lema 3.3.3.1:

$$
\eta_{B}(t) t-B(t)=\int_{0}^{t}\left[\eta_{B}(t)-\eta_{B}(u)\right] d u-c_{2}
$$

\section{Prova:}

Ver demonstração do Lema A3.1 do Apêndice A3.

Observamos que $\eta_{B}(t)$ satisfaz a seguinte equação de renovação

$$
\begin{aligned}
& \eta_{B}(t)=k(t)+\int_{0}^{t} \eta_{B}(t-x) g(x) d x \text { onde } \\
& k(t)=\eta_{A R}(t) \bar{G}(t)+c_{1} g(t) .
\end{aligned}
$$

Lema 3.3.3.2: Sejam $\hat{\eta}(s)=\int_{0}^{\infty} e^{-s t} \eta(t) d t$ a transformada de Laplace de $\eta(s), s \geq 0$ e $\hat{k}, \hat{g}$ e $\hat{\phi}$ as transformadas de Laplace de $k, g$ e $\phi$, respectivamente. Como $\eta$ satisfaz a equação de renovação, temos

$$
\hat{\eta}(s)=\frac{\hat{k}(s)}{1-\hat{g}(s)}
$$

Prova: Ver demonstração do Lema A3.2 do Apêndice A3.

Lema 3.3.3.3: Se $\phi(t)=\int_{0}^{t}[\eta(t)-\eta(u)] d u$ então

$$
\hat{\phi}(s)=-\frac{d \hat{\eta}(s)}{d s}-\frac{1}{s} \hat{\eta}(s)
$$

Prova: i) Ver demonstração do Lema A3.3 do Apêndice A3. 
Teorema 3.3.3.4: Suponhamos que $\eta$ satisfaça a equação de renovação

$$
\eta(t)=k(t)+\int_{0}^{t} \eta(t-x) g(x) d x
$$

onde $g$ é uma função densidade de probabilidade em $[0, \infty)$ e $k$ é localmente limitada. Seja $\phi(t)=\int_{0}^{t}[\eta(t)-\eta(u)] d u$ e assumamos que as seguintes quantidades são finitas:

$$
\begin{aligned}
& \mu_{i}=\int_{0}^{\infty} t^{i} g(t) d t, i=1,2, \\
& K=\int_{0}^{\infty} k(t) d t \mathrm{e} \\
& v_{l}=\frac{1}{K} \int_{0}^{\infty} t k(t) d t .
\end{aligned}
$$

Se $\phi$ é monótona, então $\phi(t) \rightarrow K\left[\frac{v_{1}}{\mu_{1}}-\frac{\mu_{2}}{2 \mu_{1}^{2}}\right]$ quando $t \rightarrow \infty$.

Prova: Ver demonstração do Teorema A3.4 do Apêndice A3.

Teorema 3.3.4.5: Assumamos que $\eta_{B}(T)$ é não decrescente em $\mathrm{T}$.

Então se $\mu_{1}=E[\zeta]$ e $\mu_{2}=E\left[\zeta^{2}\right]$ são finitos e

$$
\left(E[R(\zeta)]+c_{1}\right)\left\{\frac{\int_{00}^{\infty} \int_{0}^{y} t \phi(t, y) d t g(y) d y+c_{1} \mu_{1}}{\left(E[R(\zeta)]+c_{1}\right) \mu_{1}}-\frac{\mu_{2}}{2 \mu_{1}^{2}}\right\}>c_{2},
$$

existe $T_{0}, 0<T_{0}<\infty$, que minimiza $J_{B}(T)$. 
Prova: Do Lema 3.3.3.1 ii) e do Teorema 3.3.3.4, temos que

$$
\begin{aligned}
& L_{B}=\lim _{T \rightarrow \infty}\left[\eta_{B}(T) T-B(T)\right]=\lim _{T \rightarrow \infty}\left[\phi(T)-c_{2}\right]= \\
& =\left\{\frac{K v_{1}}{\mu_{1}}-\frac{K \mu_{2}}{2 \mu_{1}^{2}}-c_{2}\right\}=\left[K \frac{2 v_{1} \mu_{1}-\mu_{2}}{2 \mu_{1}^{2}}-c_{2}\right] .
\end{aligned}
$$

Mas

$$
\begin{aligned}
& K=\int_{0}^{\infty} k(t) d t=\int_{0}^{\infty}\left\{\eta_{A R}(t) \bar{G}(t)+c_{1} g(t)\right\} d t=c_{1}+\int_{0}^{\infty} \frac{1}{\bar{G}(t)} \int_{t}^{\infty} \phi(t, y) g(y) d y \bar{G}(t) d t= \\
& =c_{1}+\int_{0}^{\infty} \int_{t}^{\infty} \phi(t, y) g(y) d y d t=c_{1}+\int_{0}^{\infty} \int_{0}^{\infty} \phi(t, y) d t g(y) d y=c_{1}+\int_{0}^{\infty} E[R(y) / \zeta=y] g(y) d y= \\
& =c_{1}+E[R(\zeta)], \\
& \int_{0}^{\infty} t k(t) d t=\int_{0}^{\infty} t\left\{\eta_{A R}(t) \bar{G}(t)+c_{1} g(t)\right\} d t= \\
& =\int_{0}^{\infty} t\left\{\left[\frac{1}{\bar{G}(t)} \int_{t}^{\infty} \phi(t, y) g(y) d y \bar{G}(t)\right]+c_{1} g(t)\right\} d t= \\
& =\int_{0}^{\infty} t \int_{t}^{\infty} \phi(t, y) g(y) d y d t+c_{1} \int_{0}^{\infty} t g(t) d t= \\
& =\int_{0}^{\infty} y \\
& =\int_{0} t \phi(t, y) d t g(y) d y+c_{1} \mu_{1} .
\end{aligned}
$$

Concluímos que

$$
\begin{aligned}
& L_{B}=\left[K \frac{2 v_{1} \mu_{1}-\mu_{2}}{2 \mu_{1}^{2}}-c_{2}\right]= \\
& =\left[\left(E[R(\zeta)]+c_{1}\right)\left\{\frac{c_{1} \mu_{1}+\int_{00}^{\infty} \int_{0} t \phi(t, y) d t g(y) d y}{\left(E[R(\zeta)]+c_{1}\right) \mu_{1}}-\frac{\mu_{2}}{\mu^{2}{ }_{1}}\right\}-c_{2}\right]
\end{aligned}
$$


e $L_{B}>0$ se e somente se

$$
\left(E[R(\zeta)]+c_{1}\right)\left\{\frac{c_{1} \mu_{1}+\int_{0}^{\infty} \int_{0}^{y} t \phi(t, y) d t g(y) d y}{\left(E[R(\zeta)]+c_{1}\right) \mu_{1}}-\frac{\mu_{2}}{\mu^{2}{ }_{1}}\right\}>c_{2} .
$$

\section{Exemplo 3.3.4 - Política de manutenção por bloco com desconto}

Teorema 3.3.4.1: Assumamos que $\eta_{B}(T)$ seja não decrescente. Então se

$$
\left\{\frac{1}{\alpha \mu_{1}}\left(E[R(\zeta)]+c_{1}\right)-c_{2}\right\}>\frac{\left\{E\left[\alpha \int_{0}^{\infty} e^{-\alpha v} R(v \wedge \zeta) d v\right]+c_{1} E\left[e^{-\alpha \zeta}\right]\right\}}{\left[1-E\left(e^{-\alpha \zeta}\right)\right]}
$$

existe $T_{0}, O<T_{0}<\infty$, que minimiza $J_{B}^{(\alpha)}(T)$.

Prova: Neste caso, $\eta_{B}{ }^{(a)}(\infty)=\frac{1}{\alpha} \eta_{B}(\infty)-c_{2}$ e $J_{B}^{(\alpha)}(T)=\frac{B^{(\alpha)}(T)}{\left[1-e^{-\alpha T}\right]}$ com $B^{(\alpha)}(T)=\int_{[0, T)} e^{-\alpha v} A^{(\alpha)}(T-x) d U(x)$, expressão obtida no Teorema 2.1.8.

$\eta_{B}{ }^{(\alpha)}(T)$ é não decrescente pois $\eta_{B}(T)$ é não decrescente por hipótese.

Como $e^{-\alpha x} U(x)$ é a função de renovação gerada pela função de subdistribuição $H(x)=\int_{[0, x)} e^{-\alpha y} d G(y)$, concluímos que

$$
\lim _{T \rightarrow \infty} B^{(\alpha)}(T)=B^{(\alpha)}(\infty)=\frac{A^{(\alpha)}(\infty)}{\left\{1-E\left[e^{-\alpha \zeta}\right]\right\}}(\text { Feller, 1.966) }
$$

Como o numerador e o denominador da expressão de $J_{B}^{(\alpha)}(T)$ são limitados, então $L>0$ se e somente se $\eta_{B}{ }^{(\alpha)}(\infty)>J^{(\alpha)}{ }_{B}(\infty)=B^{(\alpha)}(\infty)$. Mas 


$$
\begin{aligned}
& \eta_{B}(\infty)=\lim _{T \rightarrow \infty} B^{\prime}(T)=\lim _{T \rightarrow \infty} \frac{B(T)}{T}=\lim _{T \rightarrow \infty} \frac{\int_{[0, T)} A(T-x) d U(x)}{T}= \\
& =A(\infty) \lim _{T \rightarrow \infty} \frac{\int_{[0, T)} d U(x)}{T}=\frac{A(\infty)}{\mu_{1}}=\frac{1}{\mu_{1}}\left(E\left[R(\zeta)+c_{1}\right]\right) .
\end{aligned}
$$

Assim, $L>0$ se

$$
\begin{gathered}
\eta_{B}{ }^{(\alpha)}(\infty)=\frac{1}{\alpha} \eta_{B}(\infty)-c_{2}=\frac{1}{\mu_{1}}\left(E\left[R(\zeta)+c_{1}\right]\right)-c_{2}> \\
>\frac{\left\{E\left[\alpha \int_{0}^{\infty} e^{-\alpha v} R(v \wedge \zeta) d v\right]+c_{1} E\left[e^{-\alpha \zeta}\right]\right\}}{\left[1-E\left(e^{-\alpha \zeta}\right)\right]} .
\end{gathered}
$$


Apêndice A1 
Lema A.1.1: Seja $\left\{\left(S_{n}, Z_{n}\right) ; n \geq 1\right\}$ um processo construído de acordo com a descrição do Exemplo 1.3.1. Se $N(t)=\sum_{n=1}^{\infty} I_{[0, t]}\left(S_{n}\right)$ então $\{N(t), t \geq 0\}$ é um processo de Poisson não homogêneo com função de média $\Lambda(t)=-\ln \bar{F}(t)$.

Prova: É fácil notar que $N(t)$ é uma função não decrescente e contínua à direita. Vamos calcular as distribuições finitamente-dimensionais e desenvolver a prova para o caso bidimensional. Para as outras dimensões a demonstração é semelhante.

Sejam $0 \leq t_{1} \leq t_{2}$ e $0 \leq n_{1}<n_{2}$

$$
\begin{aligned}
& P\left\{N\left(t_{1}\right)=n_{1}, N\left(t_{2}\right)=n_{2}\right\}=P\left\{S_{n_{1}} \leq t_{1}<S_{n_{1+1}} \leq S_{n_{2}} \leq t_{2}<S_{n_{2+1}}\right\}= \\
& \left.=E\left\{I_{\left\{s_{n_{1}} \leq t_{1}<s_{n_{1}+1} \leq s_{n_{2}} \leq t_{2}<s_{n_{2}+1}\right.}\right\}\right\}=\int_{0}^{t_{1}} \bar{F}^{-1}\left(s_{1}\right) \int_{s_{1}}^{t_{1}} \bar{F}^{-1}\left(s_{2}\right) . . \int_{s_{n_{1}}-1}^{t_{1}} \bar{F}^{-1}\left(s_{n_{1}}\right) \\
& \int_{t_{1}}^{t_{2}} \bar{F}^{-1}\left(s_{n_{1}+1}\right) \int_{s_{n_{1}+1}}^{t_{2}} \bar{F}^{-1}\left(s_{n_{1}+2}\right) . . \int_{s_{n_{2}-1}}^{t_{2}} \bar{F}^{-1}\left(s_{n_{2}}\right) \int_{t_{2}}^{\infty} d F\left(s_{n_{2}+1}\right) . . d F\left(s_{1}\right)= \\
& =\left[\int_{0}^{t_{1}} \bar{F}^{-1}\left(s_{1}\right) \int_{s_{1}}^{t_{1}} \bar{F}^{-1}\left(s_{2}\right) \ldots \int_{s_{n_{1}-1}}^{t_{1}} \bar{F}^{-1}\left(s_{n_{1}}\right) d F\left(s_{n_{1}}\right) \ldots d F\left(s_{1}\right)\right] \\
& {\left[\int_{t_{1}}^{t_{2}} \bar{F}^{-1}\left(s_{n_{1}+1}\right) \int_{s_{n_{1}+1}}^{t_{2}} \bar{F}^{-1}\left(s_{n_{1}+2}\right) . . \int_{s_{n_{2}-1}}^{t_{2}} \bar{F}^{-1}\left(s_{n_{2}}\right) d F\left(s_{n_{2}}\right) . . d F\left(s_{n_{1}+1}\right)\right] \bar{F}\left(t_{2}\right)=} \\
& =\left[\int_{0}^{t_{1}} \bar{F}^{-1}\left(s_{1}\right) \int_{s_{1}}^{t_{1}} \bar{F}^{-1}\left(s_{2}\right) \ldots \int_{s_{n_{1}}-2}^{t_{1}} \bar{F}^{-1}\left(s_{n_{1}-1}\right)\left(\ln \bar{F}\left(s_{n_{1}-1}\right)-\ln \bar{F}\left(t_{1}\right)\right) d F\left(s_{n_{1}-1}\right) \ldots d F\left(s_{1}\right)\right] \\
& {\left[\int_{t_{1}}^{t_{2}} \bar{F}^{-1}\left(s_{n_{1}+1}\right) \ldots \int_{s_{n_{2}-1}}^{t_{2}} \bar{F}^{-1}\left(s_{n_{2}-1}\right)\left(\ln \bar{F}\left(s_{n_{2}-1}\right)-\ln \bar{F}\left(t_{2}\right)\right) d F\left(s_{n_{2-1}}\right) \ldots d F\left(s_{n_{1}+1}\right)\right] \bar{F}\left(t_{2}\right)=}
\end{aligned}
$$




$$
\begin{aligned}
& =\left[\int_{0}^{t_{1}} \bar{F}^{-1}\left(s_{1}\right) \int_{s_{1}}^{t_{1}} \bar{F}^{-1}\left(s_{2}\right) . . \int_{s_{n_{1}-2}}^{t_{1}} \bar{F}^{-1}\left(s_{n_{1}-1}\right)\left(\ln \frac{\bar{F}\left(s_{n_{1}-1}\right)}{\bar{F}\left(t_{1}\right)}\right) d F\left(s_{n_{1}-1}\right) . . d F\left(s_{1}\right)\right] \\
& {\left[\int_{t_{1}}^{t_{2}} \bar{F}^{-1}\left(s_{n_{1}+1}\right) . . \int_{s_{n_{2}-2}}^{t_{2}} \bar{F}^{-1}\left(s_{n_{2}-1}\right)\left(\ln \frac{\bar{F}\left(s_{n_{2}-1}\right)}{\bar{F}\left(t_{2}\right)}\right) \ldots d F\left(s_{n_{2}-1}\right) d F\left(s_{n_{1}+1}\right)\right] \bar{F}\left(t_{2}\right)=} \\
& =\left[\int_{0}^{t_{1}} \bar{F}^{-1}\left(s_{1}\right) \int_{s_{1}}^{t_{1}} \bar{F}^{-1}\left(s_{2}\right) . . \int_{s_{n_{1}-3}}^{t_{1}} \bar{F}^{-1}\left(s_{n_{1}-2}\right) \frac{1}{2}\left(\ln \frac{\bar{F}\left(s_{n_{1}-2}\right)}{\bar{F}\left(t_{1}\right)}\right)^{2} d F\left(s_{n_{1}-2}\right) . . d F\left(s_{1}\right)\right] \\
& {\left[\int_{t_{1}}^{t_{2}} \bar{F}^{-1}\left(s_{n_{1}+1}\right) \ldots \int_{s_{n_{2}-3}}^{t_{2}} \bar{F}^{-1}\left(s_{n_{2}-2}\right) \frac{1}{2}\left(\ln \frac{\bar{F}\left(s_{n_{2}-2}\right)}{\bar{F}\left(t_{2}\right)}\right)^{2} \ldots d F\left(s_{n_{2}-2}\right) d F\left(s_{n_{1}+1}\right)\right] \bar{F}\left(t_{2}\right)=\ldots} \\
& =\left[\int_{0}^{t_{1}} \bar{F}^{-1}\left(s_{1}\right) \int_{s_{1}}^{t_{1}} \bar{F}^{-1}\left(s_{2}\right) \frac{1}{\left(n_{1}-2\right) !}\left(\ln \frac{\bar{F}\left(s_{2}\right)}{\bar{F}\left(t_{1}\right)}\right)^{\left(n_{1}-2\right)} d F\left(s_{2}\right) d F\left(s_{1}\right)\right] \\
& {\left[\int_{t_{1}}^{t_{2}} \bar{F}^{-1}\left(s_{n_{1}+1}\right) \int_{s_{n^{+}}}^{t_{2}} \bar{F}^{-1}\left(s_{n_{1}+2}\right) \frac{1}{\left(n_{2}-n_{1}-2\right) !}\left(\ln \frac{\bar{F}\left(s_{n_{1}+2}\right)}{\bar{F}\left(t_{2}\right)}\right)^{\left(n_{2}-n_{1}-2\right)} d F\left(s_{n_{1}+2}\right) d F\left(s_{n_{1}+1}\right)\right] \bar{F}\left(t_{2}\right)=} \\
& =\left[\int_{0}^{t_{1}} \bar{F}^{-1}\left(s_{1}\right) \frac{1}{\left(n_{1}-1\right) !}\left(\ln \frac{\bar{F}\left(s_{1}\right)}{\bar{F}\left(t_{1}\right)}\right)^{\left(n_{1}-1\right)} d F\left(s_{1}\right)\right] \\
& {\left[\int_{t_{1}}^{t_{2}} \bar{F}^{-1}\left(s_{n_{1}+1}\right) 1 \frac{1}{\left(n_{2}-n_{1}-1\right) !}\left(\ln \frac{\bar{F}\left(s_{n_{1}+2}\right)}{\bar{F}\left(t_{2}\right)}\right)^{\left(n_{2}-n_{1}-1\right)} d F\left(s_{n_{1}+1}\right)\right] \bar{F}\left(t_{2}\right)=} \\
& =\left.\left.\frac{1}{n_{1} !}\left(-\ln \frac{\bar{F}\left(s_{1}\right)}{\bar{F}\left(t_{1}\right)}\right)^{n_{1}}\right|_{0} ^{t_{1}} \frac{1}{\left(n_{2}-n_{1}\right) !}\left(-\ln \frac{\bar{F}\left(s_{n_{1}+1}\right)}{\bar{F}\left(t_{2}\right)}\right)^{\left(n_{2}-n_{1}\right)}\right|_{t_{1}} ^{t_{2}} \bar{F}\left(t_{2}\right)=
\end{aligned}
$$




$$
\begin{aligned}
& =\frac{1}{n_{1} !}\left[\left(\ln \frac{\bar{F}(0)}{\bar{F}\left(t_{1}\right)}\right)^{n_{1}}-\left(\ln \frac{\bar{F}\left(t_{1}\right)}{\bar{F}\left(t_{1}\right)}\right)^{n_{1}}\right] \\
& \frac{1}{\left(n_{2}-n_{1}\right) !}\left[\left(\ln \frac{\bar{F}\left(t_{1}\right)}{\bar{F}\left(t_{2}\right)}\right)^{\left(n_{2}-n_{1}\right)}-\left(\ln \frac{\bar{F}\left(t_{2}\right)}{\bar{F}\left(t_{2}\right)}\right)^{\left(n_{2}-n_{1}\right)}\right] \bar{F}\left(t_{2}\right)= \\
& =\frac{1}{n_{1} !}\left(-\ln \bar{F}\left(t_{1}\right)\right)^{n_{1}} \times \frac{1}{\left(n_{2}-n_{1}\right) !}\left(-\ln \bar{F}\left(t_{2}\right)-\left(-\ln \bar{F}\left(t_{1}\right)\right)\right)^{\left(n_{2}-n_{1}\right)} \bar{F}\left(t_{2}\right)= \\
& =\frac{1}{n_{1} !}\left(\Lambda\left(t_{1}\right)\right)^{n_{1}} \frac{1}{\left(n_{2}-n_{1}\right) !}\left(\Lambda\left(t_{2}\right)-\Lambda\left(t_{1}\right)\right)^{\left(n_{2}-n_{1}\right)} \exp \left\{-\Lambda\left(t_{2}\right)\right\} .
\end{aligned}
$$

Lema A.1.2: Sob as condições do Lema 1.3.2.1, temos que $L(t)=\sum_{n=1}^{N(t)} Z_{n}$, o número de reparos completos e $M(t)=N(t)-L(t)$, o número de reparos mínimos, são processos de Poisson não homogêneos independentes com médias $\int_{0}^{t} p(u) d \Lambda(u) \mathrm{e} \int_{0}^{t} q(u) d \Lambda(u)$, respectivamente.

Prova: Deveremos calcular as distribuições finitamente dimensionais. Como anteriormente, a título de ilustração será considerado somente o caso bidimensional. Sejam $0 \leq t_{1} \leq t_{2}, \quad m_{i} \quad e \quad l_{i}$ inteiros não negativos, $i=1,2 \ldots$. Definimos também $r_{0}=0$ e $r_{i}=r_{i-1}+\left(m_{i}+l_{i}\right)$, para $i=1,2$. Então

$$
\begin{aligned}
& P\left\{M\left(t_{i}\right)-M\left(t_{i-1}\right)=m_{i}, L\left(t_{i}\right)-L\left(t_{i-1}\right)=l_{i}, i=1,2\right\}= \\
& =P\left\{M\left(t_{i}\right)-M\left(t_{i-1}\right)=m_{i},\left(N\left(t_{i}\right)-M\left(t_{i}\right)\right)-\left(N\left(t_{i-1}\right)-M\left(t_{i-1}\right)\right)=l_{i}, i=1,2\right\}= \\
& =P\left\{M\left(t_{i}\right)-M\left(t_{i-1}\right)=m_{i},\left(N\left(t_{i}\right)-N\left(t_{i-1}\right)\right)-\left(M\left(t_{i}\right)-M\left(t_{i-1}\right)\right)=l_{i}, i=1,2\right\}= \\
& =P\left\{M\left(t_{i}\right)-M\left(t_{i-1}\right)=m_{i},\left(N\left(t_{i}\right)-N\left(t_{i-1}\right)\right)=l_{i}+m_{i}, i=1,2\right\}=
\end{aligned}
$$




$$
\begin{aligned}
& =P\left\{S_{r_{i}} \leq t_{i}<S_{r_{i}+1}, \sum_{v=r_{i}-1^{+1}}^{r_{i}} Z_{v+1}=l_{i}, i=1,2\right\}= \\
& =\prod_{i=1}^{2}\left[\int_{t_{i-1}}^{t_{i}} \bar{F}^{-1}\left(s_{r_{i}-1^{+1}}\right) \int_{s_{r_{i}-1^{+1}}}^{t_{i}} \bar{F}^{-1}\left(s_{r_{i}-1^{+}}\right) \cdot . \cdot \int_{s_{i_{i}-1}}^{t_{i}} \bar{F}^{-1}\left(s_{r_{i}}\right)\right. \\
& \sum_{j_{i-1}+2} \prod_{\eta_{i}+j_{r_{i}+1=l_{i}}} \prod_{v=r_{i}-1^{+2}}^{n^{+1}} p^{j_{v}}\left(s_{v-1}\right) q^{1-j_{v}}\left(s_{v-1}\right) d F\left(s_{r_{i}}\right) \ldots d F\left(s_{r_{i}-1+1}\right) \int_{t_{2}}^{\infty} d F\left(s_{r^{+}+1}\right)= \\
& =\prod_{i=1}^{2}\left[\int_{t_{i}-1}^{t_{i}} \bar{F}^{-1}\left(s_{r_{i}-1}\right) \int_{s_{r_{i}-1}+1}^{t_{i}} \bar{F}^{-1}\left(s_{r_{i}-1}+2\right) \ldots \int_{s_{r_{i}-1}}^{t_{i}} \bar{F}^{-1}\left(s_{r_{i}}\right)\right. \\
& \left.\sum_{j_{r_{i}-1+2}+\ldots+j_{r_{i}+1=l_{i}}} \prod_{v=r_{i}-1+2}^{r_{i}+1} p^{j_{v}}\left(s_{v-1}\right) q^{1-j_{v}}\left(s_{v-1}\right) d F\left(s_{r_{i}}\right) . . . d F\left(s_{r_{i}-1+1}\right)\right] \exp \left(-\Lambda\left(t_{2}\right)\right)
\end{aligned}
$$

Na expressão acima, assumimos que $O=r_{0}<r_{1}<r_{2}$. Assim, é suficiente mostrar

que

$$
\begin{aligned}
& \int_{x}^{y} \bar{F}^{-1}\left(s_{1}\right) \int_{s_{1}}^{y} \bar{F}^{-1}\left(s_{2}\right) \ldots \int_{s_{n-1}}^{y} \bar{F}^{-1}\left(s_{n}\right) \sum_{j_{2}+\ldots+j_{n-1}-l} \prod_{v=1}^{n+1} p^{j_{v}}\left(s_{v-1}\right) q^{1-j_{v}}\left(s_{v-1}\right) d F\left(s_{n}\right) . . d F\left(s_{1}\right)= \\
& =\frac{1}{l !(n-l) !}\left[\int_{x}^{y} q(u) \bar{F}^{-1}(u) d F(u)\right]^{n-l}\left[\int_{x}^{y} p(u) \bar{F}^{-1}(u) d F(u)\right]^{l},
\end{aligned}
$$

para todo $x \leq y, l=0,1,2, \ldots$ e $n=0,1, \ldots$. Para tanto, procederemos por indução.

Se $n=0$ ou $n=1$ é fácil notar que a expressão acima é verdadeira. Suponhamos que a prova seja válida para $x \leq y, l=0,1,2, \ldots$ e $n=0,1, \ldots(k-1)$ e consideremos o caso em que $n=k$. 
Se $l=0$ ou $l=k$, o resultado segue facilmente. Então, assumindo que $0<l<k$ e considerando-se os dois possíveis resultados para o primeiro ensaio, temos que

$\int_{x}^{y} \bar{F}^{-1}\left(s_{1}\right) \int_{s_{1}}^{y} \bar{F}^{-1}\left(s_{2}\right) \ldots \int_{s_{n-1}}^{y} \bar{F}^{-1}\left(s_{n}\right) \sum_{j_{2}+\ldots+j_{n-1}=1} \prod_{\nu=2}^{n+1} p^{j_{v}}\left(s_{v-1}\right) q^{1-j_{v}}\left(s_{v-1}\right) d F\left(s_{n}\right) \ldots d F\left(s_{1}\right)=$

$=\int_{x}^{y} \bar{F}^{-1}\left(s_{1}\right) p\left(s_{1}\right)\left[\int_{s_{1}}^{y} \bar{F}^{-1}\left(s_{2}\right) \ldots \int_{s_{k-1}}^{y} \bar{F}^{-1}\left(s_{k}\right) \ldots\right.$

$\left.\sum_{j_{3}+\ldots+j_{n-1}=l-1} \prod_{v=3}^{k+1} p^{j_{v}}\left(s_{v-1}\right) q^{1-j_{v}}\left(s_{v-1}\right) d F\left(s_{n}\right) \ldots d F\left(s_{2}\right)\right] d F\left(s_{1}\right)+$

$+\int_{x}^{y} \bar{F}^{-1}\left(s_{1}\right) q\left(s_{1}\right)\left[\int_{s_{1}}^{y} \bar{F}^{-1}\left(s_{2}\right) \ldots \int_{s_{k-1}}^{y} \bar{F}^{-1}\left(s_{k}\right) \ldots \sum_{j_{3}+\ldots+j_{n-1}=1} \prod_{\nu=3}^{k+1} p^{j_{v}}\left(s_{\nu-1}\right) q^{1-j_{v}}\left(s_{\nu-1}\right) d F\left(s_{n}\right) \ldots d F\left(s_{2}\right)\right] d F\left(s_{1}\right)=$

$=\int_{x}^{y} \bar{F}^{-1}(s) p(s) \frac{1}{(l-1) !(k-l) !}\left[\int_{x}^{y} q(u) \bar{F}^{-1}(u) d F(u)\right]^{k-l}\left[\int_{x}^{y} p(u) \bar{F}^{-1}(u) d F(u)\right]^{l-1} d F(s)+$

$+\int_{x}^{y} \bar{F}^{-1}(s) q(s) \frac{1}{l !(k-1-l) !}\left[\int_{x}^{y} q(u) \bar{F}^{-1}(u) d F(u)\right]^{k-1-l}\left[\int_{x}^{y} p(u) \bar{F}^{-1}(u) d F(u)\right]^{l} d F(s)=$

$=-\int_{x}^{y} \frac{d}{d F(s)}\left\{\frac{1}{l !(k-l) !}\left[\int_{s}^{y} q(u) \bar{F}^{-1}(u) d F(u)\right]^{k-l}\left[\int_{s}^{y} p(u) \bar{F}^{-1}(u) d F(u)\right]^{l}\right\} d F(s)=$

$=\frac{1}{l !(k-l) !}\left[\int_{s}^{y} q(u) \bar{F}^{-1}(u) d F(u)\right]^{k-l}\left[\int_{s}^{y} p(u) \bar{F}^{-1}(u) d F(u)\right]^{l}$. 
Lema A.1.3: O tempo entre trocas não planejadas $\zeta$ tem função de sobrevivência

$$
\bar{G}(t)=\exp \left\{-\int_{0}^{t} p(y) \bar{F}(y) d F(y)\right\} \equiv \bar{F}_{p}(t)
$$

Prova:

$$
\begin{aligned}
\bar{G}(t) & =P\{\zeta>t\}= \\
& =E\left[\prod_{i=1}^{N(t)} q\left(S_{i}\right)\right]=\sum_{n=0}^{\infty} E\left[\prod_{i=1}^{N(t)} q\left(S_{i}\right) ; N(t)=n\right]=\sum_{n=0}^{\infty} E\left[\prod_{i=1}^{n} q\left(S_{i}\right) ; S_{n} \leq t<S_{n+1}\right],
\end{aligned}
$$

Contudo,

$$
\begin{aligned}
& E\left[\prod_{i=1}^{n} q\left(S_{i}\right) ; S_{n} \leq t<S_{n+1}\right]=\sum_{z_{1} z_{2} \ldots z_{n}=0}^{1} \int_{0}^{\infty} \bar{F}^{-1}\left(y_{1}\right) \int_{y_{1}}^{\infty} \bar{F}^{-1}\left(y_{2}\right) \ldots \int_{y_{n-2}}^{\infty} \bar{F}^{-1}\left(y_{n-1}\right) \int_{y_{n-1}}^{\infty} \bar{F}^{-1}\left(y_{n}\right) \\
& \int_{y_{n}}^{\infty} \prod_{i=2}^{n+1} p\left(y_{i-1}\right)^{z_{i}} q\left(y_{i-1}\right)^{1-z_{i}} \prod_{i=1}^{n} q\left(y_{i}\right) l_{\left[y_{n} ; y_{n+1}\right]}(t) F\left(d y_{n+1}\right) \ldots F\left(d y_{1}\right)= \\
& =\int_{0}^{\infty} \bar{F}^{-1}\left(y_{1}\right) \int_{y_{1}}^{\infty} \bar{F}^{-1}\left(y_{2}\right) \ldots \int_{y_{n-2}}^{\infty} \bar{F}^{-1}\left(y_{n-1}\right) \int_{y_{n-1}}^{\infty} \bar{F}^{-1}\left(y_{n}\right) \\
& \int_{y_{n}}^{\infty}\left\{\sum_{z_{1} z_{2} \ldots z_{n}=0}^{1} \prod_{i=2}^{n+1} p\left(y_{i-1}\right)^{z_{i}} q\left(y_{i-1}\right)^{1-z_{i}}\right\} \prod_{i=1}^{n} q\left(y_{i}\right) I_{\left[y_{n} ; y_{n+1}\right]}(t) F\left(d y_{n+1}\right) \ldots F\left(d y_{1}\right) .
\end{aligned}
$$

Como estamos adicionando $\prod_{i=2}^{n+1} p\left(y_{i-1}\right)^{z_{i}} q\left(y_{i-1}\right)^{1-z_{i}}$ para todos os possíveis valores de $Z_{i}$, temos que $\sum_{z_{1}, . ., z_{n}=0}^{1} \prod_{i=2}^{n+1} p\left(y_{i-1}\right)^{z_{i}} q\left(y_{i-1}\right)^{1-z_{i}}=1$ e portanto 


$$
\begin{aligned}
& E\left[\prod_{i=1}^{n} q\left(S_{i}\right) ; S_{n} \leq t<S_{n+1}\right]=\int_{0}^{\infty} \bar{F}^{-1}\left(y_{1}\right) \int_{y_{1}}^{\infty} \bar{F}^{-1}\left(y_{2}\right) \ldots \int_{y_{n-2}}^{\infty} \bar{F}^{-1}\left(y_{n-1}\right) \int_{y_{n-1}}^{\infty} \bar{F}^{-1}\left(y_{n}\right) \\
& \int_{y_{n}}^{\infty} \prod_{i=1}^{n} q\left(y_{i}\right) I_{\left[y_{n} ; y_{n+1}\right]}(t) F\left(d y_{n+1}\right) \ldots F\left(d y_{1}\right)= \\
& =\int_{0}^{\infty} q\left(y_{1}\right) \bar{F}^{-1}\left(y_{1}\right) \int_{y_{1}}^{\infty} q\left(y_{2}\right) \bar{F}^{-1}\left(y_{2}\right) \ldots \int_{y_{n-2}}^{\infty} q\left(y_{n-1}\right) \bar{F}^{-1}\left(y_{n-1}\right) \\
& \int_{y_{n}}^{\infty} q\left(y_{n}\right) \bar{F}^{-1}\left(y_{n}\right) I_{[0 ; t]}\left(y_{n}\right) \int_{t}^{\infty} F\left(d y_{n+1}\right) \ldots F\left(d y_{1}\right)= \\
& =\bar{F}_{F}^{\infty}(t) \int_{0}^{\infty} q\left(y_{1}\right) \bar{F}^{-1}\left(y_{1}\right) \int_{y_{1}}^{\infty} q\left(y_{2}\right) \bar{F}^{-1}\left(y_{2}\right) \ldots \int_{y_{n-2}}^{\infty} q\left(y_{n-1}\right) \bar{F}^{-1}\left(y_{n-1}\right) \\
& \int_{y_{n}}^{\infty} q\left(y_{n}\right) \bar{F}^{-1}\left(y_{n}\right) I_{[0 ; t]}\left(y_{n}\right) F\left(d y_{n}\right) \ldots F\left(d y_{1}\right) .
\end{aligned}
$$

Aplicando sucessivamente o Teorema de Fubini para inverter a ordem de integração das variáveis, obtemos a seguinte expressão:

$$
\begin{aligned}
E\left[\prod_{i=1}^{n} q\left(y_{i}\right) ; Y_{n} \leq t<Y_{n+1}\right] & = \\
& =\bar{F}(t) \int_{0}^{t} q\left(y_{n}\right) \bar{F}^{-1}\left(y_{n}\right) \int_{0}^{y_{n}} q\left(y_{n-1}\right) \bar{F}^{-1}\left(y_{n-1}\right) \ldots \int_{0}^{y_{2}} q\left(y_{1}\right) \bar{F}^{-1}\left(y_{1}\right) F\left(d y_{1}\right) \ldots F\left(d y_{n}\right) .
\end{aligned}
$$

No que segue, provaremos por indução que

$$
E\left[\prod_{i=1}^{n} q\left(y_{i}\right) ; Y_{n} \leq t<Y_{n+1}\right]=\frac{\bar{F}(t)}{n !}\left[\int_{0}^{t} q\left(y_{1}\right) \bar{F}^{-1}\left(y_{1}\right) f\left(y_{1}\right) d y_{1}\right]^{n} .
$$


Se $n=2$,

$$
\begin{aligned}
& \bar{F}(t) \int_{0}^{t} q\left(y_{2}\right) \bar{F}^{-1}\left(y_{2}\right) \int_{0}^{y_{2}} q \bar{F}^{-1}\left(y_{1}\right) F\left(d y_{1}\right) F\left(d y_{n}\right)= \\
& =\bar{F}(t) \int_{0}^{t} q\left(y_{2}\right)\left(y_{2}\right) \bar{F}^{-1}\left(y_{2}\right) \int_{0}^{y_{2}} q\left(y_{1}\right) \bar{F}^{-1}\left(y_{1}\right) f\left(y_{1}\right) d y_{1} f\left(y_{2}\right) d y_{2} .
\end{aligned}
$$

Fazendo $u=\int_{0}^{y_{2}} q\left(y_{1}\right) \bar{F}^{-1}\left(y_{1}\right) f\left(y_{1}\right) d y_{1}$, temos $d u=q\left(y_{2}\right) \bar{F}^{-1}\left(y_{2}\right) f\left(y_{2}\right) d y_{2}$ e fazendo $d v=q\left(y_{2}\right) \bar{F}^{-1}\left(y_{2}\right) f\left(y_{2}\right) d y_{2}, t$ emos $v=\int_{0}^{y_{2}} q\left(y_{1}\right) \bar{F}^{-1}\left(y_{1}\right) f\left(y_{1}\right) d y_{1}$ de maneira que $\bar{F}(t) \int_{0}^{t} q\left(y_{2}\right) \bar{F}^{-1}\left(y_{2}\right) \int_{0}^{y_{2}} q\left(y_{1}\right) \bar{F}^{-1}\left(y_{1}\right) f\left(y_{1}\right) d y_{1} f\left(y_{2}\right) d y_{2}=$

$$
\begin{aligned}
& =\bar{F}(t)\left\{\left[\int_{0}^{t} q\left(y_{1}\right) \bar{F}^{-1}\left(y_{1}\right) f\left(y_{1}\right) d y_{1}\right]^{2}-\int_{0}^{t} q\left(y_{2}\right) 2 \bar{F}^{-1}\left(y_{2}\right) f\left(y_{2}\right) d y_{2} \int_{0}^{y_{2}} q 1 \bar{F}^{-1}\left(y_{1}\right) f\left(y_{1}\right) d y_{1}\right\}= \\
& =\bar{F}(t)\left[\int_{0}^{t} q\left(y_{1}\right) \bar{F}^{-1}\left(y_{1}\right) f\left(y_{1}\right) d y_{1}\right]^{2}+
\end{aligned}
$$$$
-\bar{F}(t) \int_{0}^{t} q\left(y_{2}\right) 2 \bar{F}^{-1}\left(y_{2}\right) f\left(y_{2}\right) d y_{2} \int_{0}^{y_{2}} q 1 \bar{F}^{-1}\left(y_{1}\right) f\left(y_{1}\right) d y_{1} . E
$$$$
2 \int_{0}^{t} q\left(y_{2}\right) \bar{F}^{-1}\left(y_{2}\right) \int_{0}^{y_{2}} q\left(y_{1}\right) \bar{F}^{-1}\left(y_{1}\right) f\left(y_{1}\right) d\left(y_{1}\right) f\left(y_{2}\right) d y_{2}=\left[\int_{0}^{t} q\left(y_{1}\right) \bar{F}^{-1}\left(y_{1}\right) f\left(y_{1}\right) d y_{1}\right]^{2} \text {. Assim, }
$$$$
\int_{0}^{t} q\left(y_{2}\right) \bar{F}^{-1}\left(y_{2}\right) \int_{0}^{y_{2}} q\left(y_{1}\right) \bar{F}^{-1}\left(y_{1}\right) F\left(d y_{1}\right) F\left(d y_{2}\right)=
$$$$
=\frac{1}{2}\left[\int_{0}^{t} q\left(y_{1}\right) \bar{F}^{-1}\left(y_{1}\right) f\left(y_{1}\right) d y_{1}\right]^{2} .
$$ 
Supondo a validade para $k=n-1$, temos

$$
\begin{aligned}
& \bar{F}(t) \int_{0}^{t} q\left(y_{n-1}\right) \bar{F}^{-1}\left(y_{n-1}\right) \int_{0}^{y_{n-2}} q\left(y_{n-3}\right) \bar{F}^{-1}\left(y_{n-3}\right) \ldots \int_{0}^{y_{2}} q\left(y_{1}\right) \bar{F}^{-1}\left(y_{1}\right) F\left(d y_{1}\right) \ldots F\left(d y_{n-1}\right)= \\
& =\bar{F}(t) \frac{1}{(n-1) !}\left[\int_{0}^{t} q\left(y_{n}\right) \bar{F}^{-1}\left(y_{n}\right) f\left(y_{1}\right) d y_{1}\right]^{n-1} .
\end{aligned}
$$

Vamos provar que a expressão vale para $k=n$.

$$
\begin{aligned}
& \bar{F}(t) \int_{0}^{t} q \bar{F}^{-1}\left(y_{n}\right) \int_{0}^{y_{n}} q \bar{F}^{-1}\left(y_{n-1}\right) \ldots \int_{0}^{y_{2}} q\left(y_{1}\right) \bar{F}^{-1}\left(y_{1}\right) F\left(d y_{1}\right) \ldots F\left(d y_{n-1}\right) F\left(d y_{n}\right)= \\
& =\bar{F}(t) \int_{0}^{t} q\left(y_{n}\right) \bar{F}^{-1}\left(y_{n}\right)\left[\frac{1}{(n-1) !} \int_{0}^{y_{n}} q\left(y_{1}\right) \bar{F}^{-1}\left(y_{1}\right) f\left(y_{1}\right) d y_{1}\right] f\left(y_{n}\right) d y_{n}= \\
& =\frac{\bar{F}(t)}{(n-1) !} \int_{0}^{t} q\left(y_{n}\right) \bar{F}^{-1}\left(y_{n}\right)\left[\int_{0}^{y_{n}} q\left(y_{1}\right) \bar{F}^{-1}\left(y_{1}\right) f\left(y_{1}\right) d y_{1}\right]^{n-1} f\left(y_{n}\right) d y_{n} .
\end{aligned}
$$

Fazendo $u=\left[\int_{0}^{y_{n}} q\left(y_{1}\right) \bar{F}^{-1}\left(y_{1}\right) f\left(y_{1}\right) d y_{1}\right]_{-2}^{n-1}$, temos $d u=(n-1)\left[\int_{0}^{y_{n}} q\left(y_{1}\right) \bar{F}^{-1}\left(y_{1}\right) f\left(y_{1}\right) d y_{1}\right]^{n-2} q\left(y_{n}\right) \bar{F}^{-1}\left(y_{n}\right) f\left(y_{n}\right) d y_{n} \mathrm{e}$ fazendo $d v=q\left(y_{n}\right) \bar{F}^{-1}\left(y_{n}\right) f\left(y_{n}\right) d y_{n}$, temos $v=\int_{0}^{y_{n}} q\left(y_{1}\right) \bar{F}^{-1}\left(y_{1}\right) f\left(y_{1}\right) d y_{1}$. Assim,

$$
\begin{aligned}
& \bar{F}(t) \int_{0}^{t} q \bar{F}^{-1}\left(y_{n}\right) \int_{0}^{y_{n}} q \bar{F}^{-1}\left(y_{n-1}\right) \ldots \int_{0}^{y_{2}} q\left(y_{1}\right) \bar{F}^{-1}\left(y_{1}\right) F\left(d y_{1}\right) \ldots F\left(d y_{n-1}\right) F\left(d y_{n}\right)= \\
& =\frac{\bar{F}(t)}{n !} \int_{0}^{t}\left[q\left(y_{1}\right) \bar{F}^{-1}\left(y_{1}\right) f\left(y_{1}\right) d y_{1}\right]^{n} .
\end{aligned}
$$


Portanto concluímos que

$$
\begin{aligned}
E\left[\prod_{i=1}^{N(t)} q\left(S_{i}\right)\right] & =\sum_{n=1}^{\infty} \frac{\bar{F}(t)}{n !}\left\{\int_{0}^{t} q(x) \bar{F}^{-1}(x) F(d x)\right\}^{n}= \\
& =\bar{F}(t) \exp \left\{\int_{0}^{t} q(x) \bar{F}^{-1}(x) F(d x)\right\}= \\
& =\bar{F}(t) \exp \left\{\int_{0}^{t}(1-p(x)) \bar{F}^{-1}(x) F(d x)\right\}= \\
& =\bar{F}(t) \exp \left\{\int_{0}^{t} \bar{F}^{-1}(x) F(d x)\right\} \exp \left\{-\int_{0}^{t} p(x) \bar{F}^{-1}(x) F(d x)\right\}= \\
& =\exp \left\{-\int_{0}^{t} p(x) \bar{F}^{-1}(x) F(d x)\right\} .
\end{aligned}
$$


Apêndice A2 
Lema A.2.1 : Nas condições do Teorema 2.2.1, temos

$$
\begin{aligned}
& \int e_{(0, t]}^{-\alpha \omega} d Q^{*}(\omega)=\sum_{j=1}^{k}\left[e^{-\alpha \sigma_{j-1}} R^{(\alpha)}\left(\zeta_{j}\right)+e^{-\alpha \sigma j} c_{1}\right]+e^{-\alpha \sigma k} R^{(\alpha)}\left(T-\sigma_{k}\right)+e^{-\alpha T} c_{2}, \\
& \text { onde } R_{i}^{(\alpha)}(t)=\alpha \int_{0}^{t} e^{-\alpha v} R(v+) d v+e^{-\alpha t} R_{i}(t), \text { para } \sigma_{k}<t \leq \sigma_{k+1} .
\end{aligned}
$$

Podemos pensar em $R^{(\alpha)}(t), \xi_{i} \leq t<\xi_{i+1}$ como o custo operacional com desconto exponencial da i-ésima unidade em funcionamento no intervalo de tempo $[0, t)$.

Prova: Como

$$
\begin{aligned}
& \int_{(0, t]}^{e^{-\alpha \omega}} d Q^{*}(\omega)=\alpha \int_{0}^{t} e^{-\alpha \omega} Q^{*}(\omega) d \omega+\left(\left.e^{-\alpha \omega} Q^{*}(\omega)\right|_{0} ^{t}=\right. \\
& =\alpha \int_{0}^{t} e^{-\alpha \omega} Q^{*}(\omega) d \omega+e^{-\alpha t} Q^{*}(t)-Q^{*}(0),
\end{aligned}
$$

podemos escrever que, para $\sigma_{k}<T \leq \sigma_{k+1}$,

$$
\begin{aligned}
& \int_{(0, T]} e^{-\alpha \omega} d Q^{*}(\omega)=\alpha \int_{0}^{T} e^{-\alpha \omega} Q^{*}(\omega) d \omega+e^{-\alpha T} Q^{*}(T)-Q^{*}(0)= \\
& =\alpha \int_{0}^{T} e^{-\alpha \omega} Q^{*}(\omega) d \omega+e^{-\alpha T} Q^{*}(T)= \\
& =e^{-\alpha T}\left\{\sum_{i=1}^{k}\left(R\left(\zeta_{i}\right)+c_{1}\right)+R\left(T-\sigma_{k}\right)+c_{2}\right\}+ \\
& +\alpha \sum_{j=1}^{k} \int_{\sigma-1}^{\sigma} e^{-\alpha \omega}\left\{\sum_{i=1}^{j-1} R\left(\zeta_{i}\right)+c_{1}+R\left(\omega-\sigma_{j-1}\right)\right\} d \omega+ \\
& +\alpha \int_{\sigma_{k}}^{T} e^{-\alpha \omega}\left\{\sum_{i=1}^{k} R\left(\zeta_{i}\right)+c_{1}+R\left(\omega-\sigma_{k}\right)\right\} d \omega .
\end{aligned}
$$


Mas

$$
\begin{aligned}
& \alpha \sum_{j=1}^{k} \int_{\sigma_{j-1}}^{\sigma j} e^{-\alpha \omega}\left\{\sum_{i=1}^{j-1} R\left(\zeta_{i}\right)+c_{1}+R\left(\omega-\sigma_{j-1}\right)\right\} d \omega= \\
& =\alpha \sum_{j=1}^{k} \int_{\sigma_{j-1}}^{\sigma_{j}} e^{-\alpha \omega}\left\{\sum_{i=1}^{j-1} R\left(\zeta_{i}\right)+c_{1}+\right\} d \omega+\alpha \sum_{j=1}^{k} \int_{\sigma_{j-1}}^{\sigma_{j}} e^{-\alpha \omega} R\left(\omega-\sigma_{j-1}\right) d \omega= \\
& =\alpha\left\{\sum_{i=1}^{j-1} R\left(\zeta_{i}\right)+c_{1}+\right\} \sum_{j=1}^{k} \int_{\sigma_{j-1}}^{\sigma_{j}} e^{-\alpha \omega} d \omega+\alpha \sum_{j=1}^{k} \int_{\sigma_{j-1}}^{\sigma_{j}} e^{-\alpha \omega} R\left(\omega-\sigma_{j-1}\right) d \omega= \\
& =\alpha\left\{\sum_{i=1}^{j-1} R\left(\zeta_{i}\right)+c_{1}+\right\} \sum_{j=1}^{k}\left(e^{-\alpha \sigma_{j-1}}-e^{-\alpha \sigma_{j}}\right)+\alpha \sum_{j=1}^{k} \int_{\sigma_{j-1}}^{\sigma_{j}} e^{-\alpha \omega} R\left(\omega-\sigma_{j-1}\right) d \omega,
\end{aligned}
$$

$\mathrm{e}$

$$
\begin{aligned}
& a \sum_{j=1}^{k} \int_{\sigma_{k}}^{T} e^{-a \omega}\left\{\sum_{i=1}^{j-1} R\left(\zeta_{i}\right)+c_{1}+R\left(\omega-\sigma_{k}\right)\right\} d \omega= \\
& =a \int_{\sigma_{k}}^{T} e^{-a \omega}\left\{\sum_{i=1}^{k} R\left(\zeta_{i}\right)+c_{1}\right\} d \omega+a \int_{\sigma_{k}}^{T} e^{-a \omega} R(\omega-\sigma k) d \omega= \\
& =\left\{\sum_{i=1}^{k} R\left(\zeta_{i}\right)+c_{1}\right\}\left(e^{-a \sigma_{k}}-e^{-a T}\right)+a \int_{\sigma_{k}}^{T} e^{-a \omega} R\left(\omega-\sigma_{k}\right) d \omega .
\end{aligned}
$$

Então

$$
\begin{aligned}
\int_{(0, T]}^{e^{-\alpha \omega}} d \omega & =e^{-\alpha T} \sum_{i=1}^{k}\left(R\left(\zeta_{i}\right)+c_{1}\right)+e^{-\alpha T} R\left(T-\sigma_{k}\right)+e^{-\alpha T} c_{2}+ \\
& +\sum_{j=1}^{k}\left(e^{-\alpha \sigma j-1}-e^{-\alpha \sigma}\right)\left(\sum_{i=1}^{j-1} R\left(\zeta_{i}\right)+c_{1}\right)+\alpha \sum_{j=1}^{k} \int_{\sigma_{j-1}}^{\sigma_{j}} e^{-\alpha \omega} R\left(\omega-\sigma_{j-1}\right) d \omega+ \\
& +e^{-\alpha \sigma_{k}} \sum_{i=1}^{k}\left(R\left(\zeta_{i}\right)+c_{1}\right)+\alpha \int_{\sigma_{k}}^{T} e^{-\alpha \omega} R\left(\omega-\sigma_{k}\right)-e^{-\alpha T} \sum_{i=1}^{k}\left(R\left(\zeta_{i}\right)+c_{1}\right) .
\end{aligned}
$$

Fazendo $v=\omega-\sigma_{j-1}$, temos $d v=d \omega, \omega==v+\sigma_{j-1} \mathrm{e}$ 


$$
\begin{aligned}
& \int_{(0, T]}^{e^{-\alpha \omega}} d \omega=e^{-\alpha T} R\left(T-\sigma_{k}\right)+e^{-\alpha T} c_{2}+ \\
& +\sum_{j=1}^{k}\left(e^{-\alpha \sigma_{j-1}}-e^{-\alpha \sigma_{j}}\right)\left(\sum_{i=1}^{j-1} R\left(\zeta_{i}\right)+c_{1}\right)+\alpha \sum_{j=1}^{k} \int_{0}^{\sigma_{j}-\sigma_{j-1}} e^{-\alpha\left(v+\sigma_{j-1}\right)} R(v) d v+ \\
& +e^{-\alpha \sigma_{k}} \sum_{i=1}^{k}\left(R\left(\zeta_{i}\right)+c_{1}\right)+\alpha \int_{0}^{T-\sigma_{k}} e^{-\alpha\left(v+\sigma_{k}\right)} R(v) d v= \\
& =\sum_{j=1}^{k}\left(e^{-\alpha \sigma_{j}-1}-e^{-\alpha \sigma_{j}}\right)\left(\sum_{i=1}^{j-1} R\left(\zeta_{i}\right)+c_{1}\right)+e^{-\alpha \sigma k} \sum_{i=1}^{k}\left(R\left(\zeta_{i}\right)+c_{1}\right)+ \\
& +\alpha \sum_{j=1}^{k} e^{-\alpha \sigma_{j-1}} \int_{0}^{\zeta_{j}} e^{-\alpha \nu} R(v) d v+\alpha e^{-\alpha \sigma_{k}} \int_{0}^{T-\sigma_{k}} e^{-\alpha v} R(v) d v+ \\
& +e^{-\alpha\left(T-\sigma_{k}\right)} e^{-\alpha \sigma k} R\left(T-\sigma_{k}\right)+e^{-\alpha T} c_{2}= \\
& =\sum_{i=0}^{k-1} \sum_{j=i+1}^{k}\left(e^{-\alpha \sigma j-1}-e^{-\alpha \sigma j}\right)\left(R\left(\zeta_{i}\right)+c_{1}\right)+e^{-\alpha \sigma k} \sum_{i=1}^{k}\left(R\left(\zeta_{i}\right)+c_{1}\right)+ \\
& +\alpha \sum_{j=1}^{k} e^{-\alpha \sigma_{j-1}} \int_{0}^{\zeta j} e^{-\alpha v} R(v) d v+\alpha e^{-\alpha \sigma_{k}} \int_{0}^{T-\sigma_{k}} e^{-\alpha v} R(v) d v+ \\
& +e^{-\alpha\left(T-\sigma_{k}\right)} e^{-\alpha \sigma_{k}} R\left(T-\sigma_{k}\right)+e^{-\alpha T} c_{2}= \\
& =\sum_{i=0}^{k-1}\left(e^{-\alpha \sigma i}-e^{-\alpha \sigma k}\right)\left(R\left(\zeta_{i}\right)+c_{1}\right)+e^{-\alpha \sigma k} \sum_{i=1}^{k}\left(R\left(\zeta_{i}\right)+c_{1}\right)+ \\
& +\alpha \sum_{j=1}^{k} e^{-\alpha \sigma_{j-1}} \int_{0}^{\zeta j} e^{-\alpha v} R(v) d v+\alpha e^{-\alpha \sigma_{k}} \int_{0}^{T-\sigma_{k}} e^{-\alpha v} R(v) d v+ \\
& +e^{-\alpha\left(T-\sigma_{k}\right)} e^{-\alpha \sigma_{k}} R\left(T-\sigma_{k}\right)+e^{-\alpha T} c_{2}=
\end{aligned}
$$




$$
\begin{aligned}
& =-e^{-\alpha \sigma_{k}} \sum_{i=0}^{k-1}\left(R\left(\zeta_{i}\right)+c_{1}\right)+e^{-\alpha \sigma k} \sum_{i=0}^{k}\left(R\left(\zeta_{i}\right)+c_{1}\right)+ \\
& +\sum_{i=0}^{k-1} e^{-\alpha \sigma_{i}}\left(R\left(\zeta_{i}\right)+c_{1}\right)+\alpha \sum_{j=1}^{k} e^{-\alpha \sigma} j-1 \int_{0}^{\zeta j} e^{-\alpha v} R(v) d v+ \\
& +e^{-\alpha \sigma_{k}} \alpha \int_{0}^{T-\sigma_{k}} e^{-\alpha \nu} R(v) d v+e^{-\alpha\left(T-\sigma_{k}\right)} e^{-\alpha \sigma_{k}} R\left(T-\sigma_{k}\right)+e^{-\alpha T} c_{2}= \\
& =e^{-\alpha, \sigma_{k}}\left(R\left(\zeta_{k}\right)+c_{1}\right)+\sum_{i=0}^{k-1} e^{-\alpha \sigma_{i}}\left(R\left(\zeta_{i}\right)+c_{1}\right)+ \\
& +\alpha \sum_{j=1}^{k} e^{-\alpha \sigma j-1} \int_{0}^{\zeta j} e^{-\alpha v} R(v) d v+e^{-\alpha \sigma} k R^{(\alpha)}\left(T-\sigma_{k}\right)+e^{-\alpha T} c_{2}= \\
& =\sum_{i=0}^{k} e^{-\alpha \sigma_{i}}\left(R\left(\zeta_{i}\right)+c_{1}\right)+\alpha \sum_{j=1}^{k} e^{-\alpha \sigma j-1} \int_{0}^{\zeta_{j}} e^{-\alpha v} R(v) d v+ \\
& +e^{-\alpha \sigma_{k}}\left\{\alpha \int_{0}^{T-\sigma_{k}} e^{-\alpha v} R(v) d v+e^{-\alpha\left(T-\sigma_{k}\right)} R\left(T-\sigma_{k}\right)\right\}+e^{-\alpha T} c_{2}= \\
& =\sum_{i=1}^{k} e^{-\alpha\left(\zeta_{i}+\sigma_{i-1}\right)}\left(R\left(\zeta_{i}\right)+c_{1}\right)+ \\
& +\sum_{i=1}^{k} e^{-\alpha \sigma_{i-1}} \alpha \int_{0}^{\zeta_{i}} e^{-\alpha v} R(v) d v+e^{-\alpha \sigma_{k}} R^{(\alpha)}\left(T-\sigma_{k}\right)+e^{-\alpha T} c_{2}= \\
& =\sum_{i=1}^{k}\left\{e^{-\alpha\left(\zeta_{i}+\sigma_{i-1}\right)} c_{1}+e^{-\alpha \sigma_{i-1}}\left[\alpha \int_{0}^{\zeta_{i}} e^{-\alpha v} R(v) d v+e^{-\alpha \zeta_{i}}\left(R\left(\zeta_{i}\right)+c_{1}\right)\right]\right\}+ \\
& +e^{-\alpha \sigma_{k}} R^{(\alpha)}\left(T-\sigma_{k}\right)+e^{-\alpha T} c_{2}= \\
& =\sum_{i 1}^{k}\left[e^{-\alpha \sigma_{i-1}} R^{(\alpha)}\left(\zeta_{i}\right)+e^{-\alpha \sigma_{i}} c_{1}\right]+e^{-\alpha \sigma_{k}} R^{(\alpha)}\left(T-\sigma_{k}\right)+e^{-\alpha T} c_{2} .
\end{aligned}
$$


Lema A.2.2: Nas condições do Teorema 2.1.1 podemos escrever $A^{(\alpha)}(T)$ da seguinte maneira:

$$
A^{(\alpha)}(T)=E\left[\left(e^{-\alpha \zeta} c_{1}+R^{(\alpha)}(\zeta)\right) ; \zeta<T\right]+E\left[\left(e^{-\alpha T} c_{2}+R^{(\alpha)}(T)\right) ; \zeta \geq T\right]
$$

\section{Prova:}

$$
\begin{aligned}
& A^{(\alpha)}(T)=E\left[\int_{(0, \delta]} e^{-\alpha \omega} d R^{*}(\omega)\right]=E\left[e^{-\alpha \eta} R^{*}(\delta)+\alpha \int_{(0, \eta]} e^{-\alpha \omega} R^{*}(\omega) d \omega\right]= \\
& =E\left[e^{-\alpha \delta} R^{*}(\delta)+\alpha \int_{(0, \delta]} e^{-\alpha \omega} R^{*}(\omega) d \omega\right]= \\
& =E\left[\left(e^{-\alpha \zeta} R^{*}(\zeta)+\alpha \int_{(0, \zeta]} e^{-\alpha \omega} R^{*}(\omega) d \omega\right) ; \zeta<T\right]+ \\
& +E\left[\left(e^{-\alpha T} R^{*}(T)+\alpha \int_{(0, T]} e^{-\alpha \omega} R^{*}(\omega) d \omega\right) ; \zeta \geq T\right] \\
& =E\left[\left(e^{-\alpha \zeta}\left(R(\zeta)+c_{1}\right)+\alpha \int_{(0, \zeta]} e^{-\alpha \omega}\left(R(\zeta)+c_{1}\right) d \omega\right) ; \zeta<T\right]+ \\
& +E\left[\left(e^{-\alpha T}\left(R(T)+c_{2}\right)+\alpha \int_{(0, T]} e^{-\alpha \omega}\left(R(T)+c_{2}\right) d \omega\right) ; \zeta \geq T\right]= \\
& =E\left[\left(e^{-\alpha \zeta} c_{1}+R^{(\alpha)}(\zeta)\right) ; \zeta<T\right]+E\left[\left(e^{-\alpha T} c_{2}+R^{(\alpha)}(T)\right) ; \zeta \geq T\right]
\end{aligned}
$$


Apêndice A3 


\section{Lema A3.1:}

$$
\eta_{B}(t) t-B(t)=\int_{0}^{t}\left[\eta_{B}(t)-\eta_{B}(u)\right] d u-c_{2}
$$

Prova:

$$
\begin{aligned}
& \eta_{B}(t) t-B(t)=B^{\prime}(t) t-B(t)=B^{\prime}(t) t-\int_{0}^{t} B^{\prime}(u) d u-B(0)= \\
& =\int_{0}^{t} B^{\prime}(t) d u-\int_{0}^{t} B^{\prime}(u) d u-B(0)=\int_{0}^{t}\left[\eta_{B}(t)-\eta(u)\right] d u-B(0)= \\
& \int_{0}^{t}\left[\eta_{B}(t)-\eta_{B}(u)\right] d u-c_{2} .
\end{aligned}
$$

Lema A3.2: Seja $\hat{\eta}_{B}(s)=\int_{0}^{\infty} e^{-s t} \eta_{B}(t) d t$ a transformada de Laplace de $\eta_{B}(t)$. Similarmente, $\hat{k}, \hat{g}$ e $\hat{\phi}$ as transformações de Laplace de $k, g$ e $\phi$ respectivamente. Como $\eta_{B}$ satisfaz a equação de renovação

$$
\begin{aligned}
& \eta_{B}(t)=k(t)+\int_{0}^{t} \eta_{B}(t-x) g(x) d x, \text { temos } \\
& \hat{\eta}_{B}(s)=\frac{\hat{k}(s)}{1-\hat{g}(s)} .
\end{aligned}
$$

Prova: Note que

$$
\begin{aligned}
& \hat{\eta}(s)=\hat{k}(s)+\int_{0}^{\infty} e^{-s y} \int_{0}^{t} \eta(t-x) g(x) d x d y=\hat{k}(s)+\hat{\eta}(s) \hat{g}(s) \text {. Assim, } \\
& (1-\hat{g}(s)) \hat{\eta}(s)=\hat{k}(s) \text { e } \hat{\eta}(s)=\frac{\hat{k}(s)}{(1-\hat{g}(s))} .
\end{aligned}
$$


Lema A3.3: Se $\phi(t)=\int_{0}^{t}\left[\eta_{B}(t)-\eta_{B}(u)\right] d u$, então

$$
\hat{\phi}(s)=-\frac{d \hat{\eta}(s)}{d s}-\frac{1}{s} \hat{\eta}(s)
$$

Prova:

$$
\begin{aligned}
& \hat{\phi}(s)=\int_{0}^{\infty} e^{-s t} \phi(t) d t=\int_{0}^{\infty} e^{-s t} \int_{0}^{t}\left\{\eta_{B}(t)-\eta_{B}(u)\right\} d u d t= \\
&=\int_{0}^{\infty} e^{-s t} \int_{0}^{t} \eta_{B}(t) d u d t-\int_{0}^{\infty} e^{-s t} \int_{0}^{t} \eta_{B}(u) d u d t= \\
&=\int_{0}^{\infty} e^{-s t} \eta_{B}(t) t d t-\int_{0}^{\infty} \eta_{B}(u) \int_{u}^{\infty} e^{-s t} d t d u= \\
&=\int_{0}^{\infty} e^{-s t} \eta_{B}(t) t d t-\int_{0}^{\infty} \eta_{B}(u)\left[-\left.\frac{e^{-s t}}{s}\right|_{u} ^{\infty} d u=\right. \\
&=\int_{0}^{\infty} e^{-s t} \eta_{B}(t) t d t-\frac{1}{s} \int_{0}^{\infty} \eta_{B}(u) e^{-s u} d u= \\
&=\int_{0}^{\infty} e^{-s t} \eta_{B}(t) t d t-\frac{1}{s} \hat{\eta}_{B}(s) . \\
& \text { Contudo, } \frac{d \eta(s)}{d s}=\frac{d \int_{0}^{\infty} e^{-s t} \eta_{B}(t) d t}{d s}=\int_{0}^{\infty} \frac{d e^{-s t}}{d s} \eta_{B}(t) d t=\int_{0}^{\infty} e^{-s t} t \eta_{B}(t) d t
\end{aligned}
$$

concluímos a demonstração. 
Teorema A3.4: Suponhamos que $\eta$ satisfaça a equação de renovação

$$
\eta(t)=k(t)+\int_{0}^{t} \eta(t-x) g(x) d x
$$

onde $g$ é uma função densidade de probabilidade em $[0, \infty)$ e $k$ é localmente limitado. Seja $\phi(t)=\int_{0}^{t}[\eta(t)-\eta(u)] d u$ e assumamos que as seguintes quantidades são finitas:

$$
\begin{aligned}
& \mu_{i}=\int_{0}^{\infty} t^{i} g(t) d t, i=1,2, \\
& K=\int_{0}^{\infty} k(t) d t \mathrm{e} \\
& v_{1}=\frac{1}{K} \int_{0}^{\infty} t k(t) d t .
\end{aligned}
$$

Se $\phi(t)$ é monótonona, então

$$
\phi(t) \rightarrow K\left[\frac{v_{1}}{\mu_{1}}-\frac{\mu_{2}}{2 \mu_{1}^{2}}\right] \text { quando } t \rightarrow \infty .
$$

Prova: Como $\phi(t)$ é monótona, o comportamento de $\phi(t)$ quando $t \rightarrow \infty$ é determinado pelo comportamento de $s \hat{\phi}(s)$ quando $s \downarrow 0$.

$$
\begin{aligned}
& s \hat{\phi}(s)=\left\{\left[-\frac{d}{d s} \hat{\eta}(s)-\frac{1}{s} \hat{\eta}(s)\right]=-s\left[\frac{d \frac{\hat{k}(s)}{(1-\hat{g}(s))}}{d s}\right]-\hat{\eta}(s)=\right. \\
& =s\left[\frac{-\frac{d \hat{k}(s)}{d s}(1-\hat{g}(s))+\hat{k}(s) \frac{d(1-\hat{g}(s))}{d s}}{(1-\hat{g}(s))^{2}}\right]-\frac{\hat{k}(s)}{(1-\hat{g}(s))}= \\
& =\frac{-\frac{d \hat{k}(s)}{d s}}{\frac{(1-\hat{g}(s))}{s}}-\frac{\frac{\hat{k}(s)}{s} \frac{d \hat{g}(s)}{d s}}{\left[\frac{(1-\hat{g}(s))}{s}\right]^{2}}-\frac{\frac{\hat{k}(s)}{s} \frac{(1-\hat{g}(s))}{s}}{\left[\frac{(1-\hat{g}(s))}{s}\right]^{2}}=
\end{aligned}
$$




$$
=\frac{-\frac{d \hat{k}(s)}{d s}}{\frac{(1-\hat{g}(s))}{s}}-\left\{\frac{\frac{\hat{k}(s)}{s}\left[\frac{d \hat{g}(s)}{d s}+\frac{(1-\hat{g}(s))}{s}\right]}{\left[\frac{(1-\hat{g}(s))}{s}\right]^{2}}\right\} \text {. }
$$

Quando $s \downarrow 0$,

$$
\begin{aligned}
& \lim _{s \downarrow 0} \frac{(1-\hat{g}(s))}{s}=\lim _{s \downarrow 0} \int_{0}^{\infty} \frac{1-e^{-s t}}{s} g(t) d t=\int_{0}^{\infty} \int_{0}^{t} \lim _{s \downarrow 0} e^{-s u} d u g(t) d t=\int_{0}^{\infty} t g(t) d t=\mu_{1}, \\
& \lim _{s \downarrow 0} \hat{k}(s)=\int_{0}^{\infty} \lim _{s \downarrow 0} e^{-s t} k(t) d t=\int_{0}^{\infty} k(t) d t=K, \\
& \lim _{s \downarrow 0}-\frac{d \hat{k}(s)}{d s}=\lim _{s \downarrow 0} \int_{0}^{\infty} t e^{-s t} k(t) d t=K v_{1} \mathrm{e} \\
& \lim _{s \downarrow 0} \frac{1}{s}\left[\frac{d \hat{g}(s)}{d s}+\frac{(1-\hat{g}(s))}{s}\right]=\lim \left[-\int_{0}^{\infty} t \frac{e^{-s t} g(t)}{s} d t+\int_{0}^{\infty} \int_{0}^{t} \frac{e^{-s u} d u}{s} g(t) d t\right]= \\
& \lim _{s \downarrow 0}\left[\int_{0}^{\infty}\left[\int_{0}^{t} \frac{e^{-s u}}{s}-\frac{e^{-s t}}{s} d u\right) g(t) d t\right]=\lim _{s \downarrow 0}\left[\int_{0}^{\infty} \int_{0}^{t} \int_{u}^{t} e^{-s v} d v d u g(t) d t\right]= \\
& =\int_{0}^{\infty} \int_{0}^{t} \int_{u}^{t} d v d u g(t) d t=\int_{0}^{\infty} \int_{0}^{\infty}(t-u) d u g(t) d t=\int_{0}^{\infty} \frac{t^{2}}{2} g(t) d t=\frac{\mu_{2}}{2} .
\end{aligned}
$$

Consequentemente, $s \hat{\phi}(s) \rightarrow \frac{K v_{1}}{\mu_{1}}-\frac{K \mu_{2}}{2 \mu_{1}^{2}}$, quando $s \downarrow 0$. 


\section{Bibliografia}

[1] Barlow, Hunter and Proschan (1.965). "Mathematical Theory of Reliability". John Wiley \& Son, Inc.. NY.

[2] Chen and Savits (1.988). A discounted cost relationship. Technical Report, Department of Mathematics and Statistics, University of Pittsburg.

[3] Chen and Savits (1.989). Optimal age and block replacement for a general maintenance model. Technical Report, Department of Mathematics and Statistics, University of Pittsburg.

[4] Feller (1.986). "An Introduction to Probability Theory and Its Applications", Vol. II, John Willey \& Sons, Inc.. NY.

[5] Puri and Singh (1.985). Optimum replacement of a system subject to shocks: a mathematical lemma. Operation Research, 34,5, p. 782-789.

[6] Savits (1.988). A cost relationship between age and block replacemente policies. J. Appl. Prob. 25, 789-796. 